Portland State University

PDXScholar

1971

\title{
Mössbauer studies of stable interstitial and substitutional sites of cobalt in gold
}

Charles Freeland Steen

Portland State University

Follow this and additional works at: https://pdxscholar.library.pdx.edu/open_access_etds

Part of the Physics Commons

Let us know how access to this document benefits you.

\section{Recommended Citation}

Steen, Charles Freeland, "Mössbauer studies of stable interstitial and substitutional sites of cobalt in gold" (1971). Dissertations and Theses. Paper 1917.

https://doi.org/10.15760/etd.1916

This Thesis is brought to you for free and open access. It has been accepted for inclusion in Dissertations and Theses by an authorized administrator of PDXScholar. Please contact us if we can make this document more accessible: pdxscholar@pdx.edu. 
AN ABSTRACT OF THE WHESIS OF Charles Freeland Steen for the Master of Science in Physics presented May 26, 1971.

Title: Mössbauer Studies of Stable Interstitial and Substitutional Sites of Cobalt in Gold.

APPROVED BY MEMBERS OF THE THESIS COMMITTEE:

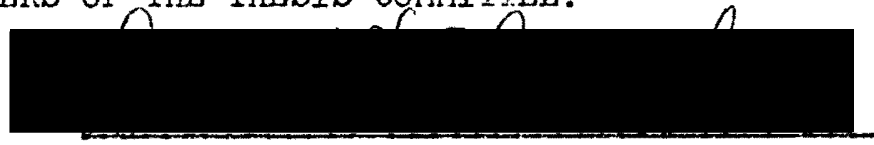

Donald G. Howard, Chairman

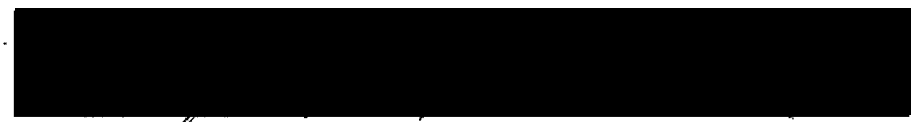

Rudi H. Nussbaum

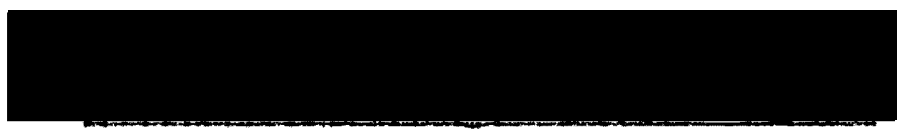

Mark Gurevitch
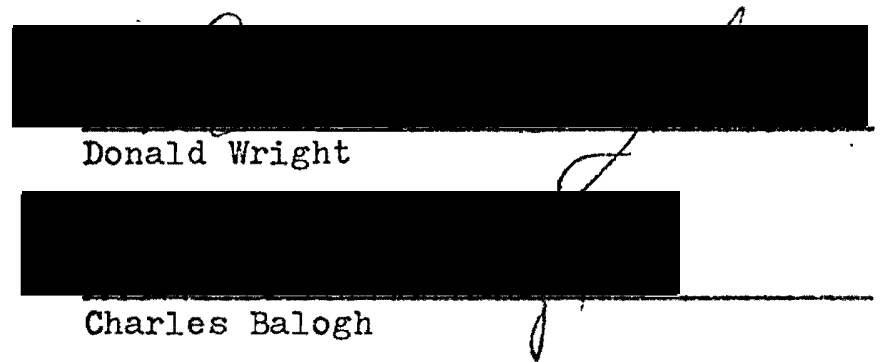

Precision measurements of the Debye-Waller factor of $\mathrm{Fe}^{57}$ in $\mathrm{Au}$ using the vide black absorber technique have been made in the temperan ture range from room temperature to $830 \mathrm{~K}$. The major source of error arises from the Au $I_{\gamma} x$-rays which are unresolved by the radiation detection system from the $14.4 \mathrm{keV}$ gamma ray. The $\mathrm{K}$ absorption edge of Br has been used to correct for the presence of the x-rays.

These measurements have revealed the existence of two nonequivalent lattice sjtes which the $\mathrm{Co}^{57}$ impurity (parent of $\mathrm{Fe}^{57}$ ) may occupy. One of the sites has been populated by quenching the source from temp 
eratures greater than $1150 \mathrm{~K}$ and has been identified as a substitution-. al lattice site. From an analysis of the Mössbauer spectrum it is concluded that some of the substitutional sites may be in the vicinity of dislocations. It has been determined that the impurity-host force constant for $\mathrm{Fe}^{57}$ atoms in the substitutional site is about the same as the host-host force constant; the experimental value of the anharmonicity parameter $\varepsilon(-2)$ for $\mathrm{Fe}^{5 ?}$ in Au. is nearly the same as calculated values of $\varepsilon(-2)$ for pure $A u$.

The second site forms very slowly upon annealing the source at intermediate temperatures and has been identified as being interstitial in character. The slow rate of formation of the interstitial site indicates that diffusion is a primary mechanism in its formation. The second site must therefore be more complicated than an ordinary interstitial. It is proposed that this site is a split $\langle 100\rangle$, or dumbbell, interstitial formed by substituting two impurity atoms for one of the Au atoms. 
MÖSSBAUER STUDIES OF STABLE INTERSTITIAL AND

SUBSTITUTIONAI, SITES OF COBALT IN GOLD

\author{
by \\ CHARLES FREELAND STEEN
}
A thesis submitted in partial fulfillment of the requirements for the degree of

MASTER OF SCIENCE

in

PHYSICS

Portland State University

1971 
TO THE OFFICE OF GRADUATE STUDIES:

The members of the Committee approve the thesis of

Charles Freeland Steen presented May 26, 1971.

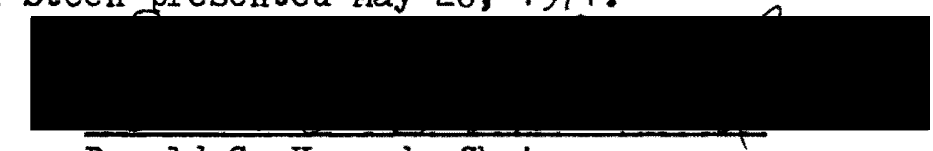

Donald G. Howard, Chairman

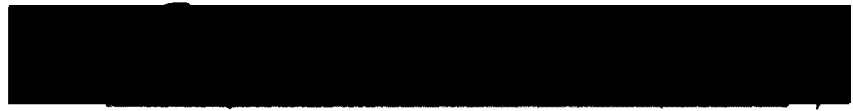

Rudi H. Nussbaum

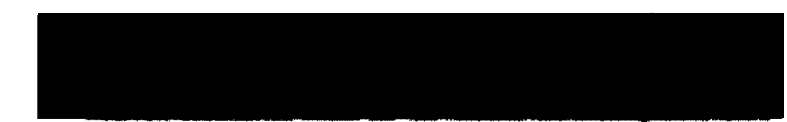

Mark Gurevitch

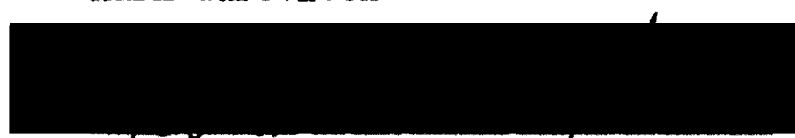

Donald Wright
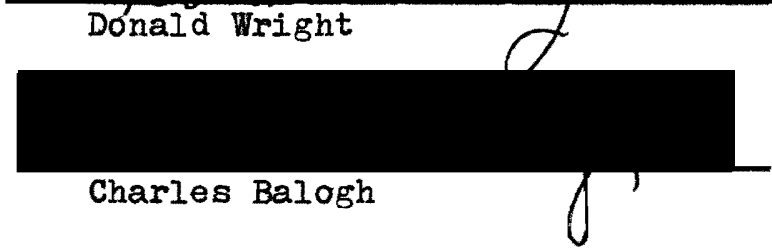

APPROVED:

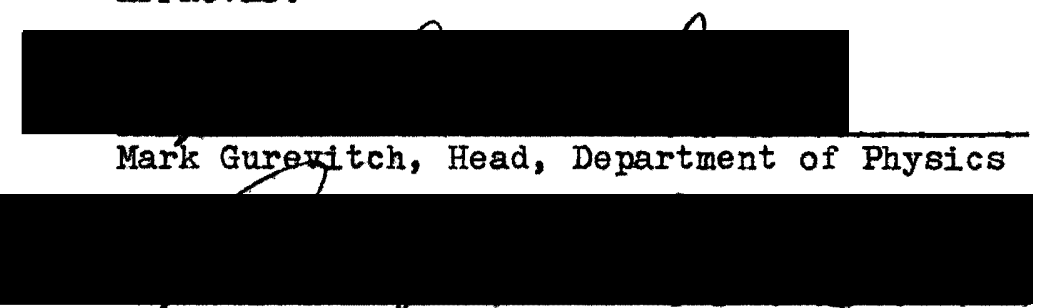

David T. Clark, Dean of Graduate Studies

June 3, 1971 


\section{ACKNOWLEDGMENTS}

This author wishes to thank the Tektronix Foundation for its fellowship and NSF for its support. My many thanks to my thesis committee, in particular to Professors D. G. Howard, R. H. Nussbaum and D. Wright for their many helpful suggestions and dicussions. Last but not least I wish to thank my wife Barbara for her patience and understanding while this thesis was being written. 
TABLE OF CONTENTS

PAGE

ACKNOWLEDGMENTS .................. . . . . . . . iii

IIST OF TABLES . . . . . . . . . . . . . . . . vi

IIST OF FIGURES . • . . . . . . . . . . . . . . . vii

INTRODUCTION . . . . . . . . . . . . . . . . 1

THEORY . . . . . . . . . . . . . . . . . . 3

I DEBYE-WALLER FACIOR IN THE HARMONIC APPROXIMATION. • 3

II ANHARMONIC CORRECTIONS TO THE DEBYE-WALLER FACTOR. • 8

III IINE SHAPE . . . . . . . . . . . . . 12

EXPERIMENTAL .......................... 16

I LINE SHAPE MEASUREMENTS . . . . . . . . 16

II SOURCE PREPARATION . . . . . . . . . . . 20

Electrolytic Deposition ........... 20

Copper .................... . . . . . 25

Diffusion of Gold ............. 27

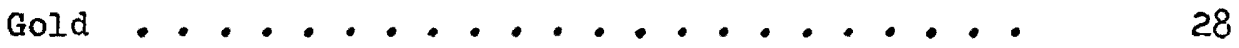

III X-RAY CORRECTION . . . . . . . . . . . 32

IV $f$ MEASUREMENTS ................ 44 
PAGE

DATA PRESENTATION AND INTERPRETATION

I SUBSTITUTIONAI SITE $\ldots . . . . . . . . .$. 50

II INTERSTITIAL SITE $\cdot . \cdot \cdot \cdot \cdot \cdot \cdot \cdot \cdot \cdot \cdot \cdot \cdot \cdot \cdot$

SUMMARY AND CONCLUSION : . . . . . . . . . . . 62

REFERENCES ......... . . . . . . . . . . . 


\section{LIST OF TABLES}

TABLE

PAGE

I Diffusion Process Used to Make Au III . . . . . .

II Absorber Parameters ................

II Transmission Coefficients and Normalized Intensities

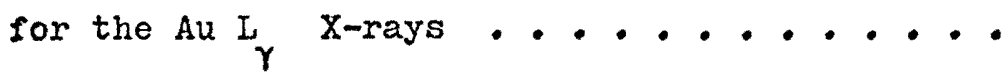

IV Iine Shape Parameters of $\mathrm{Fe}^{5 ?}$ in $\mathrm{Au}$ for Quenched and

Annealed Sources ..............

$v$ Comparison of Line Broadening with the Lattice

Parameter of the Host Lattice for Pd, Pt, and Au Sources . . . . . . . . . . . .

VI Lattice Dynamical Parameters for $\mathrm{Fe}^{5 ?}$ in $\mathrm{Au}$ for the Substitutional Lattice Site . . . . . . 


\section{LIST OF FIGURES}

1 Basic experimental setup used to make line shape measurements. . . . . . . . . . .

2 Electrolytic cell. ................

3 Source mount. . . . . . . . . . . . . .

4 Results of the electrodeposition of $\mathrm{Co}^{57}$ onto copper and gold. The solid curve is the result that Dèzsi obtains for copper. . . . . . . .

5 Heating rate of the surface of a Au sample placed inside the vycor tube. The furnace temperature

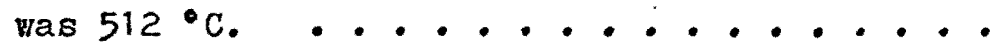

6 Energy spectrum of $\mathrm{Fe}^{57}$ in $\mathrm{Au}$. It was made using a $\mathrm{Xe}-\mathrm{CH}_{4}$ filled proportional counter, an FET preamp and a 512 channel analyzer. The crosshatched portion of the $14.4 \mathrm{keV}$ peak indicates a typical window setting of the single channel analyzer during measurements of $f$ and line

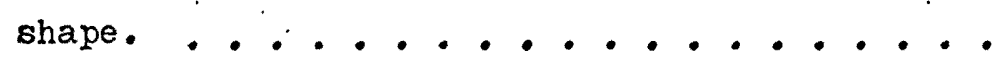


7 Line shape of Au IV made using the mechanical drive and one of the inner lines of an iron absorber. The measurement was made after the second diffusion and after the surface had been etched. The solid line is a Lorentzian curve centered about $0.20 \mathrm{~mm} / \mathrm{sec}$ with a full width at half maximum of $0.235 \mathrm{~mm} / \mathrm{sec}$. . . . .

8 Experimental setup used to determine the $x$-ray correction.

$9 \mathrm{~K}$ absorption edge of $\mathrm{Br} . . . . . . . . . .$. 40

10 Axially symmetric furnace used for the high temperature.

$f$ measurements. Saureisen is used to insulate the thermocouple wires and for potting the heater windings.

11 Temperature dependence of $(-\ln f)$ for $A u$ III, Au IIIB, and $A u$ IV. The solid symbols indicate that the source temperature was being increased between measurements; the open symbols indicate that the source temperature was being decreased between measurements. The solid lines indicate the expected behavior, equation (23). . . .

12. Time dependence of the Debye-Waller factor of Au IV at $765 \mathrm{~K}$ and $829 \mathrm{~K}$. .......... . 
13 Line shape of Au IV taken on the speaker drive using one of the lines of a nitroprusside absorber; the velocity scale has been adjusted to give velocities relative to an iron absorber. The spectrum was taken after the completion of the $f$ measurements. The asymmetry of the quadrupole split soublet is due to approximately $2.5 \%$ of the $\mathrm{Fe}$ impurities which are still in substitutional sites. ...........

14 Position of the impurity dumbbell in the Au lattice. The symmetry axis of the dumbbell can lie along any of the three [100] directions. . . 
INTRODUCTION

It is possible for a radioactive atom to emit a gamma ray without exchanging energy with the lattice vibrations of the crystal in which It is embedded. This process is called the Mössbauer effect and the probability that such an event will occur is usually referred to as the Debye-Waller factor.

The Debye-Walier factor of $\mathrm{Fe}^{57}$ in Au has been measured by several different investigators ${ }^{1-5}$. There is a genral lack of agreement in the results of these investigations. It was felt that the inconsis.. tency of these measurements was due to the methods used to prepare the Au sources. Therefore, the initial motivation of this work was to make precision measurements of the Debye-Waller factor of $\mathrm{Fe}^{57}$ in $\mathrm{Au}$ with special emphasis on the technique of source preparation.

It was found from these measurements that there are two distinct lattice sites which the Fe impurity atoms can.occupy in the Au lattice, a substitutional site and an interstitial site. We were able to observe the diffusion of the $F e$ atoms from the substitutional site into the interstitial site. Combining this information with the derived lattice dynamical parameters of the two sites, (see below), and measurements of the gamma ray spectrum, or line shape, we have concluded that the interstitial site is a split $\langle 100\rangle$, or dumbbell, 
Interstitial formed by substituting two impurity atoms for one of the $\mathrm{Au}$ atoms.

This thesis is organized into three main sections: Theory, experimental, and interpretation of data. In the theory section the theory which relates the Debye-Waller factor to lattice dynamical parameters is outlined. The factors which effect the line shape are also discussed. The emphasis of the experimental section is on the technique of source preparation (electrolytic deposition), and the use of a $\mathrm{NaBr}$ absorber to obtain the correction factor for the Au $I_{y} x$-ray (this correction arises because the $I_{\gamma} x$-ray is not resolved from the $14.4 \mathrm{keV} \mathrm{Fe} \mathrm{F}^{57}$ gamma ray). In the interpretation section the data is presented and lattice dynamical and line shape parameters are extracted for the two sites. 
THEORY

\section{DEBYE-WALLER FACTOR IN THE HARMONIC APPROXIMATION}

Consider the case of gamma ray emission from a nucleus bound in a crystal lattice. In general, the energy released during the nuclear transition is carried away by three mechanisms: the gamma ray, the recoil motion of the crystal, and the generation of phonons in the lattice. Compared to the energy of the gamma ray the recoil energy of the crystal is negligible. Furthermore, for low energy gamma rays it is possible, due to the quantized nature of the phonons, that no phonon will be generated during the emission; a process known as zero phonon emission, or the Mossbauer effect. Neglecting the recoil energy of the entire crystal, these Mössbauer gamma rays will receive all of the energy released by the nuclear transition. The probability that zero phonon emission will occur is usually called the Debye-Waller factor or the recoil free fraction.

Lipkin ${ }^{6}$ has investigated the transition probability for gamma ray emission from an atom bound in a crystal lattice. He finds that the probability that the emission will cause a transition from stationary lattice state $|I\rangle$ to stationary state $|F\rangle$ is given by

$$
P(F, I)=|\langle F|\exp (\underset{\sim}{i K} \cdot \underset{\sim j}{X})| I\rangle|^{2}
$$


where $\underset{\sim}{K}$ is the gamma ray vave vector and $\underset{\sim}{\mathrm{X}}$ is the displacement vector of the emitting atom from its equilibrium position. The probability for zero phonon emission, i.e. the case for which $|F\rangle=|I\rangle$, is given by $P(I, I)$. For any temperature other than absolute zero the initial state of the lattice is unknown, therefore, assuming thermal equilibrium, the Debye-Waller factor, $f$, is given by the thermal average of $P(I, I)$

$$
f=\frac{\sum_{n} \exp \left(-\beta E_{n}\right)|\langle I|\exp (\underset{\sim}{\operatorname{K}} \cdot \underset{\sim}{X})| I\rangle|^{2}}{\sum_{n} \exp \left(-\beta E_{n}\right)},
$$

where $B=1 / k_{B} T$ and $k_{B}$ is Boltzman's constant.

For the case of a harmonic lattice the Debye-Waller factor takes on a simpler form 7,8

where

$$
f=\exp \left[-(2 W)_{H}\right]
$$

$$
(2 W)_{H}=\left\langle[\underset{\sim}{K} \cdot \underset{\sim j}{X}]^{2}\right\rangle=K^{2}\left\langle x_{j l}^{2}\right\rangle
$$

$x_{j l}$ represents the component of $\underset{\sim j}{X}$ in the direction of $\underset{\sim}{K}$ and $\langle A\rangle$ denotes the thermal average of operator A. Housley and Hess ${ }^{9}$ have evaluated $\left\langle x_{j l}^{2}\right\rangle$ for a general harmonic lattice. They find that in thermal equilibrium

$$
\left\langle x_{j 1}^{2}\right\rangle=\frac{\pi}{m_{j}} \sum_{n} b_{j l n}^{2}\left[\frac{1}{2}+\frac{1}{\exp \left(\beta \hbar \omega_{n}\right)-1}\right] \frac{1}{\omega_{n}},
$$

where $\omega_{n}$ is the frequency of the $n^{\text {th }}$ normal mode. The $b_{j l n}$ are the components of the unitary transformation connecting $x_{j l}$ with the normal coordinates; they give the strength of the contribution of the $n^{\text {th }}$ normal mode.

In a real crystal the normal modes are close enough together 
that the sum in equation (5) may be replaced by an integral

$$
\left\langle x_{j I}^{2}\right\rangle=\frac{\pi}{m} \int_{j}^{\infty}\left[\frac{1}{2}+\frac{1}{\exp (\beta \hbar \omega)-1}\right] \frac{\mathrm{G}_{j I}(\omega)}{\omega} \mathrm{d} \omega \text {. }
$$

The function $G_{j I}(\omega) d \omega$ takes the place of $b_{j l n}^{2}$ in the sum and it is normalized such that

$$
\int_{0}^{\infty} G_{j 1}(\omega) d \omega=1
$$

If the lattice is monatomic $G_{j l}(\omega)$ is the usual density of states function ${ }^{10}$. If, as is usually the case in Mossbauer studies, the emitting atom is an impurity in an otherwise monatomic lattice $G_{j l}(\omega)$ is the dynamic response function ${ }^{11}$ which represents the coupling of the impurity atom to the frequency spectrum of the host lattice.

The value of the Debye-Waller factor cannot be obtained using the above equations unless $G_{j l}(\omega)$ is known. Since the shape of the frequency spectrum is seldom known from experiment, a form of $G_{j l}(\omega)$ must be assumed. Traditionally the Debye model ${ }^{10}$ has been used for this purpose. In this model the frequency spectrum is assumed to be proportional to $\omega^{2}$, a property which is rigorously true in the vicinIty of $\omega \rightarrow 0$ for all solids ${ }^{12}$. The constant of proportionality is determined by using the normalization condition, equation (7). For a cubic lattice

$$
G_{j I}^{D}(\omega)=\left\{\begin{array}{cc}
\left.3 \omega^{2} / \omega\right)_{D}^{3}, w \leqslant \omega_{D} \\
0, w>\omega_{D}
\end{array}\right.
$$

where $w_{b}$ is the maximum frequency which can be propagated through the Debye solid. The exponent of the Debye-Waller factor can now be written 


$$
\begin{aligned}
(2 W)_{D} & =\frac{3 K^{2} \hbar}{m_{j}{ }_{D}^{3}} \int_{0}^{\omega}\left[\frac{1}{2}+\frac{1}{\exp \left(\beta^{h} \omega\right)-T}\right] \omega d \omega \\
& =\frac{6 R}{\theta_{D} k_{B}}\left[\frac{1}{4}+\left(\frac{T}{\theta_{D}}\right)^{2} \int_{0}^{\theta_{D} / T} \frac{x d x}{e^{X}-1}\right]
\end{aligned}
$$

where $R=K^{2} r^{2} / 2 m_{j}$ and $\theta_{D}$ is the characteristic Debye temperature defined by

$$
\pi \omega_{D}=k_{B} \theta_{D}
$$

The integral in equation (9) has been tabulated for various values of $\theta_{D} / T$ by Muir $^{13}$.

There are two problems associated with using the Debye model. First, it is not a realistic model. Real frequency spectra show pronounced structure ${ }^{14}$. The only reason it fits the data reasonably well is that the Debye-Waller factor is only integrally sensitive to the shape of the real frequency spectrum with strong weighting toward the low frequencies (see equation $(6)$ ). Second, it is a one parameter model. This creates the usual problem that the fit parameter $\theta_{D}$ is temperature dependent.

Since the Debye-Waller factor is insensitive to the exact shape of the frequency spectrum the $f$ measurements cannot be used to directly determine $G_{j I}(\omega)$. Instead, the indirect approach described below must be used. Since the measurements of this investigation were carried out at elevated temperatures the high temperature expansion of equation (6) for a real harmonic crystal ${ }^{9}$ will be useful 


$$
\begin{aligned}
\left\langle x_{j I}^{2}\right\rangle=\frac{1}{\beta m_{j}} \int_{0}^{\infty}[1 & +\frac{1}{12}(\beta \hbar \omega)^{2}-\frac{1}{720}(\beta \hbar \omega)^{4} \\
& +\ldots] \frac{G_{j l}(\omega)}{\omega} d \omega
\end{aligned}
$$

This expansion converges for $\beta$ h $\omega_{\max }<2 \pi . \omega_{\max }$, like $\omega_{b}$, is the maximum frequency which can be propagated through the real lattice. This expansion can be put into a more useful form by using the frequency moments defined by

$$
\begin{aligned}
& v_{j I}(n)=\left[\int_{0}^{\infty} \omega{ }^{n_{G}}(\omega) d \omega\right]^{1 / n}, n \neq 0 \\
& v_{j I}(0)=0
\end{aligned}
$$

Equation (11) can now be written

$$
\left\langle x_{j 1}^{2}\right\rangle=\frac{1}{\beta m_{j}}\left[\frac{1}{v_{j I}(-2)}\right]^{2}+\frac{1}{12} \frac{\hbar^{2}}{\beta m_{j}}+\ldots
$$

The first term in this expansion contributes more than $95 \%$ for $\mathrm{k}_{\mathrm{B}} \mathrm{T} / \hbar v_{j I}(-2)>0.72^{15}$. To this approximation the exponent of the Debye-Wailer factor at high temperatures can be written

$$
(2 W)_{H} \approx \frac{K^{2}}{\beta m_{j}}\left[\frac{1}{v_{j l}(-2)}\right]^{2}=\frac{2 R T}{k_{B}}\left[\frac{1}{\theta_{j l}(-2)}\right]^{2},
$$

Where $\theta_{j l}(n)$ is a characteristic temperature parameter related to the moments $v_{j 1}(n)$ by

$$
\text { If } v_{j I}(n)=k_{B} \dot{\theta}_{j I}(n)
$$

In principle, if enough of these moments can be obtained with high precision from experiments then it is possible to invert equation (12) and to obtain the shape of the frequency spectrum.

Comparison between the Debye model and the expansion in frequency moments of the real crystal can be made defining the 
the equivalent Debye spectrum $G_{j I}^{D}(\omega, n)$ which gives the correct values for the moments $\nu_{j I}(n)$

$$
G_{j I}^{D}(\omega, n)=\left\{\begin{array}{cl}
\frac{3 \omega^{2}}{\omega_{D}(n)}, & \omega \leqslant \omega_{D}(n) \\
0, & \omega>\omega_{D}(n)
\end{array}\right.
$$

Using equations (12) and (16) gives

$$
v_{j 1}(n)=\left[\frac{3}{n+3}\right]^{1 / n} \omega_{D}(n)
$$

or

$$
\theta_{j I}(n)=\left[\frac{3}{n+3}\right]^{1 / n} \theta_{D}(n)
$$

where the weighted Debye temperature $\theta_{D}(n)$ is defined by

$$
\text { \# } \omega_{D}(n)=k_{B} \theta_{D}(n)
$$

For a given harmonic crystal the weighted Debye parameters $\theta_{D}(n)$ are different from each other and independent of the temperature.

At high temperatures the Debye-Waller factor depends primarily on $\theta_{D}(-2)$ as can be seen by comparing equations (14) and (18). At very low temperatures the Debye-Waller factor depends primarily on $\theta_{D}(-1)^{15}$. Therefore, a fit of experimental data to a single parameter Debye model will in general result in a temperature dependent Debye temperature $\theta_{D^{-}}$The same effect occurs for Debye temperatures derived from lattice specific heat measurements.

\section{ANHARMONIC CORRECTIONS TO THE DEBYE-WALLER FACTOR}

The harmonic model considered above is only an approximation. Forces in real crystals have anharmonic contributions, as is evidenced 
for example by the existence of thermal expansion. As will be seen, the effect of this anharmonicity is to introduce an additional temperature dependence into the Debye-Waller factor.

In obtaining equation (2) the assumption was made that the lifetime of the lattice states was much longer than the lifetime of the excited nuclear state. Anharmonicity will cause the lifetime of the lattice states to be much shorter than the nuclear lifetime ${ }^{16}$. In this case the matrix element should be squared after the thermal averaging instead of before ${ }^{17}$

$$
\sqrt{f}=\left\langle\exp \left(i x_{j} \cdot x_{j}\right)\right\rangle
$$

In order to carry out the thermal average, the shape of the anharmonic potential must be known. Maradudin and Flinn 8 , assuming nearest neighbor interactions only, have expanded the potential in a Taylor series and carried out the thermal averaging for the usual case when only the cubic and quartic anharmonic terms of the expansion are retained. They find that to a good approxiamation

$$
f=\exp \left[-(2 W)_{A H}\right]
$$

where

$$
(2 W)_{A H}=\left\langle\left[\underset{\sim}{K} \cdot X_{j}\right]^{2}\right\rangle
$$

They have evaluated $\left\langle\left[\underset{\sim}{K} \cdot \sim_{j}\right]^{2}\right\rangle$ for the case of a face centered cubic lattice and find that the exponent of the Debye-Waller factor is given by

$$
(2 W)_{A H}=(2 W)_{H}[1+\varepsilon T]
$$

where 


$$
\varepsilon=k_{B}\left\{0.3508 \frac{\left[\phi^{(3)}\left(\underset{\sim}{\sim_{0}}\right)\right]^{2}}{\left[\phi^{(2)}(\underset{\sim 0}{r})\right]^{3}}-0.25 \frac{\phi^{(4)}\left(\underset{\sim 0}{\sim_{0}}\right)}{\left[\phi^{(2)}(\underset{\sim 0}{r})\right]^{2}}\right\},
$$

$\emptyset^{(n)}\left(\underline{r}_{0}\right)$ is the $n^{\text {th }}$ derrivative of the potential evaluated at $\underline{r}_{0}$, and $\mathfrak{r}_{0}$ is the position of the potential minimum at absolute zero. $\varepsilon$ is usually referred to as the anharmonicity parameter.

Another approach to the question of anharmonicity is the quasiharmonic approximation. In this approximation the crystal is considered to be harmonic but is allowed to expand as the temperature is Increased, causing the shape of the interatomic potential to change. However, the basic assumption of the quasi-harmonic approximation is that the potential can still be considered to be harmonic and, therefore, the Debye-Waller factor is still given by equation (13) ${ }^{18}$. As the shape of the harmonic potential changes the frequency spectrum also changes. This change in the frequency spectrum due to volume expansion is described by the Grüneisen parameter defined by ${ }^{10}$

$$
\gamma=-\frac{d\left(\ln w_{D}\right)}{d(\ln V)}
$$

For a real crystal in the quasi-harmonic model we can define the weighted Grüneisen parameters $\gamma_{j l}(n)$ describing the volume dependence of the frequency moments $v_{j I}(n)$

$$
\begin{aligned}
r_{j I}(n) & =-\frac{d\left(\operatorname{In} v_{j I}(n)\right)}{d(I n V)} \\
& =-\frac{v}{v_{j I}(n)} \frac{d\left(v_{j I}(n)\right) / d T}{d V / d T}
\end{aligned}
$$

Since $(1 / V)(d V / d T)=3 \alpha$, where $\alpha$ is the Iirear expansion coefficient, 
this equation can be rewritten as

$$
\gamma_{j l}(n)=-\frac{1}{3 \alpha v_{j I}(n)} \frac{d v_{j I}(n)}{d T}
$$

Integration gives the frequency moments as a function of temperature

$$
\left[v_{j l}(n)\right]_{T}=\left[v_{j l}(n)\right]_{0} \exp \left[-3 \bar{\alpha} \gamma_{j l}(n) T\right],
$$

where

$$
\bar{\alpha}=(1 / T) \int_{0}^{T} \alpha\left(T^{\prime}\right) d T^{\prime}
$$

In the high temperature Iimit the quasi-harmonic approximation gives for the exponent of the Debye-Waller factor

$$
\text { (2W) } \begin{aligned}
{ }_{Q H} & =\frac{K^{2}}{\beta m_{j}}\left(\left[v_{j l}(-2)\right]_{T}\right)^{-2} . \\
& =\frac{K^{2}}{\beta m_{j}}\left(\left[v_{j 1}(-2)\right]_{0}\right)^{-2} \exp \left[6 \bar{\alpha} \gamma_{j 1}(-2) T\right]
\end{aligned}
$$

Expanding the exponential and keeping only the first order correction term gives back equation (23) for (2W) $\mathrm{QH}$ with

$$
\varepsilon(-2)=6 \bar{\alpha} \gamma_{j 1}(-2)
$$

It should be noted that the above relation between $\varepsilon$ and $\gamma$ is valid only in the quasi-harmonic approximation. This approximation assumes that the temperature dependence of the frequency moments arises only from volume expansion. Comparison of neutron scattering experiments with the quasi-harmonic approximation suggests that, due to anharmonicity, the frequency moments also have an explicit temperature dependence; it arises from the change in force constants due to the change with temperature of the average energy per mode ${ }^{15}$.

Pathak and Deo ${ }^{19}$ have used the results of Maradudin and Flinn ${ }^{8}$, 
equation (18), to replace the quasi-harmonic approximation by a more rigorous treatment. Starting from equation (18) they show that in the high temperature limit the exponent of the Debye-Waller factor can be written

$$
\text { (2W) }{ }_{A H}=\frac{K^{2}}{\beta m_{j}}\left[v_{j I}(-2)\right]^{-2}
$$

This result is general for an anharmonic crystal in which the phonon Iifetimes are very short compared to the lattice vibrational periods. If the (-2) moments for the real crystal are known (these can be determined from neutron scattering experiments) then the Debye- Waller factor can be calculated using equation (32); otherwise, a model must be assumed for the real crystal. If the Grüneisen parameter is used to describe the temperature dependence of the frequency moments and (2W) ${ }_{\mathrm{AH}}$ is evaluated from equation $(32), \varepsilon(-2)$ will again be given by equation (31). It should be noted, however, that the validity of equation (31) will no longer be dependent on the validity of the quasiharmonic approximation; it is only dependent on the accuracy with which the Grineisen parameter describes the temperature dependence of the lattice modes.

\section{IIJ. LINE SHAPE}

As is vell known, the energy of the emitted gamma ray is not precisely defined. The spectral distribution of the gamma rays (the number of gamma rays emitted with energy in the interval between $\mathrm{E}$ and $E+d E$ ) has a Lorentzian shape. According to the uncertainty principle, the full width at half maximum, $\Gamma$, of the gamma ray spectrum 
is given by

$$
\Gamma=\hbar / \tau
$$

where $\tau$ is the lifetime of the nuclear state. The gamma ray spectrum from a single transition is called the line shape and $\Gamma$ is referred to as the natural line width of the gamma ray.

If the gamma ray spectrum would always be a single line having natural width, then line shape measurements would only yield direct information on the nuclear lifetime. In practice, the gamma ray spectrum is often composed of more than one natural width line. From the shape of the spectrum it is possible to obtain information about the physical state of the emitting nucleus and about the chemical state of the emitting atom.

The position of the gamma ray line depends on the energy of the excited state. If the nuclear states have hyperfine structure due to internal fields there will result a gamma ray, and hence a line having natural width, from each allowable transition between hyperfine levels. If the energy difference between the hyperfine transitions is much greater than natural width, the spectrum will consist of a number of discrete lines all having natural width. If the energy difference is less than natural width the spectrum will appear to be a single line whose width is broader than natural.

One mechanism which can produce hyperfine structure in the nuclear states is the nuclear quadrupole interaction. To see how this arises consider the case of a nonspherical nucleus in an electric field gradient. The potential energy due to the interaction between the charge distribution in the nucleus and the electric field gradient is 
given by

$$
\tau=-\frac{1}{2} \sum_{i j} q_{i j} \frac{\partial E_{i}}{\partial x_{j}}
$$

where $q_{i j}$ are the components of the quadrupole moment tensor. The possible energy states are quantized; the number of possible states will depend on the magnitude of the nuclear spin. For the case of $\mathrm{Fe} 57$ the ground state has a spin of $1 / 2$ and has no quadrupole interaction. The $14.4 \mathrm{keV}$ excited state has a spin of $3 / 2$ and has two possible energy levels in an electric field gradient. Therefore, the resulting hyperfine pattern will consist of two lines. The separation of the two lines vill be proportional to the strength of the electric field gradient.

An electric field gradient cannot exist if the emitting nucleus is located at a lattice site having full cubic symmetry. The existence of quadrupole splitting therefore means that the symmetry of the lattice at the site of the nucleus is less than cubic. It should be pointed out that if the emitting atom is an impurity the local symmetry about the impurity need not be related to the symmetry of the host lattice. Clustering of the impurity atoms, occupation of interstitial rather than subsitutional sites, and other related effects can result in quadrupole splitting in host lattices which are cubic. Another closely related cause of hyperfine structure is the nuclear Zeernan effect. In this case it is the interaction of the nuclear magnetic moment with the magnetic field at the site of the nucleus (the field may be generated by a net polarization of the surrounding atomic electrons, the conduction electrons, and the rest of 
the lattice) which produces the hyperfine structure. The decay of the $14.4 \mathrm{keV} 3 / 2$ - state to the $1 / 2$ - ground state of $\mathrm{Fe}^{57}$ is by magnetic dipole radiation 20 . Therefore, there are six allowed transitions giving rise to six lines in the gamma ray spectrum, (in the absence of an electric field gradient).

The position of the gamma ray lines is also effected by the chemical state of the emitting atom 20,21 . This chemical shift (also referred to as the isomer shift) represents the electric monopole interaction, i.e. the elctrostatic interaction of a finite size charge distribution with the finite s-electron density inside the nuclear volume. The interaction energy contains a nuclear factor (the nuclear radius) and an atomic factor (the s-electron density at the nucleus). If the emitting atoms are not all in equivalent lattice sites they may have different s-electron densities at the nucleus, and hence different chemical shifts; therefore, there may be more than one line in the gamma ray spectrum. The energy differences due to chemical shifts between the different lattice sites is usually small enough that the individual lines cannot be resolved. The composit line will be broader than natural and may appear to be asymmetric. 
EXPERIMENTAL

\section{LINE SHAPE MEASUREMENTS}

The natural width of the $14.4 \mathrm{keV}$ transition of $\mathrm{Fe}^{57}$ is $4.6 \times 10^{-9} \mathrm{eV}$. Therefore, in order to measure the shape of the line an energy resolution of better than one part in $10^{-12}$ is needed. The technique used to obtain this resolution is described below.

Consider the setup shown in figure 1. Gamma rays are created in the source from the decay of the $14.4 \mathrm{keV}$ excited state of $\mathrm{Fe}^{57}$. These gamma rays are incident on $\mathrm{Fe}^{57}$ nuclei bound in the lattice of the absorber. Let the transition energy of the source be $\mathrm{E}_{e}$ and the transition energy of the absorber be $E_{a}{ }^{*}$. Then, providing there is no phonon generation during the absorption process, the gamma ray will be resonantly absorbed if

$$
E_{\gamma}=E_{a}+E_{r}
$$

where $E_{\gamma}$ is the energy of the gamma ray and $E_{r}$ is the recoil energy that must be supplied to the crystal in order to conserve momentum. The energy that the zero phonon gamma ray receives in the source is

Usually, the emitting and absorbing nuclei are in different environments. The difference in s-electron densities and internal fields of the different environments will cause the energy levels of the source to differ from those of the absorber: 


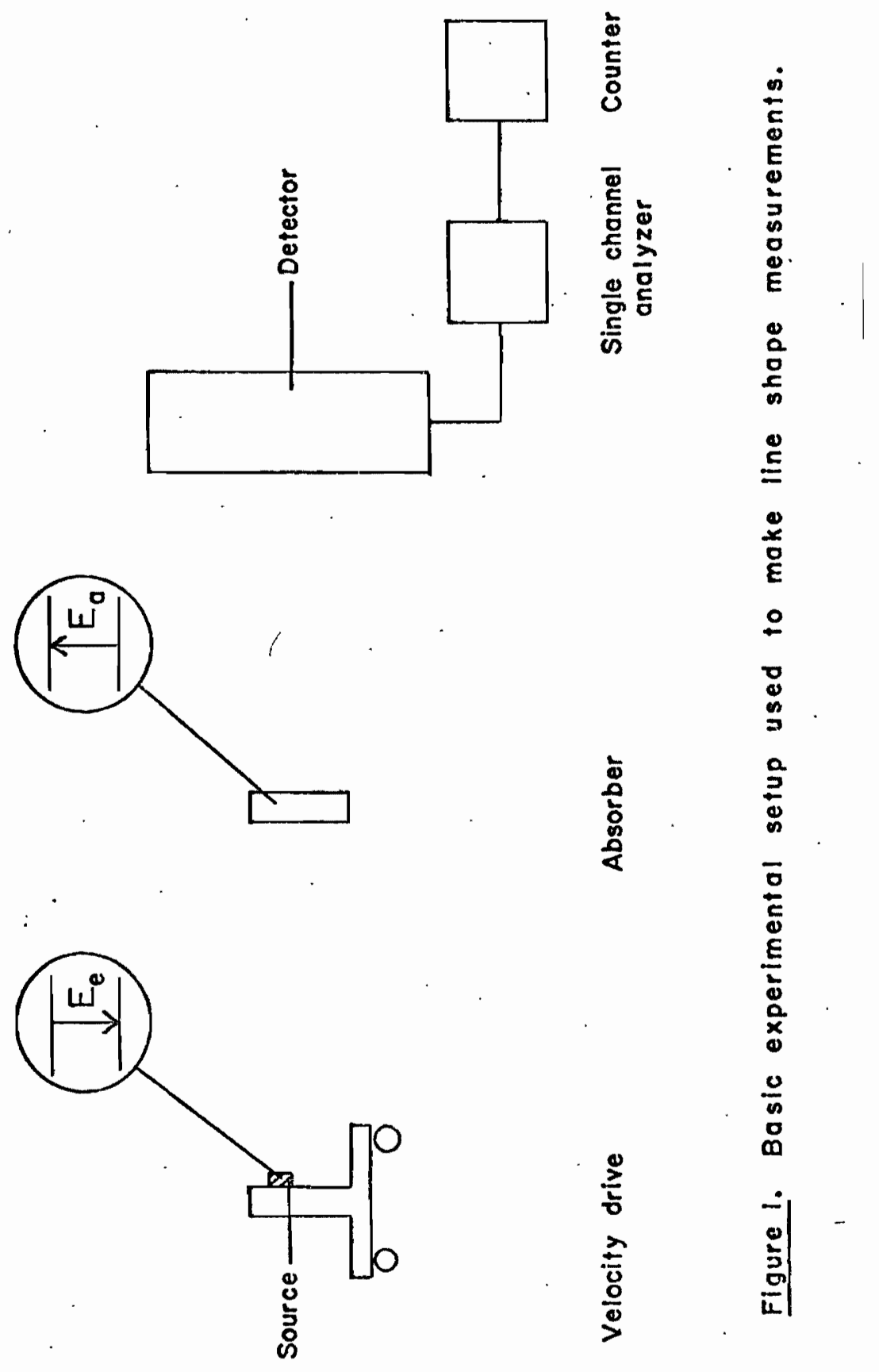




$$
E_{r}=E_{e}-E_{r}
$$

$E_{x}$ is much less than the natural width of the gamma ray and is therefore negligible. Hence, resonant absorption of the zero phonon gamma ray will take place if $E_{a}$ is equal to $E_{e}$.

If $E_{e}$ is not equal to $E_{a}$, the Doppler effect can be used to produce the condition for resonant absorption. If the source and absorber are moving with a velocity. $v$ with respect to each other the gamma ray will be Doppler shifted; its energy at the absorber will be, assuming that $v \ll c$,

$$
E_{Y}=E_{e}\left[1+\frac{v}{c}\right]
$$

$v$ is chosen as positive is the source moves toward the absorber and negative if it moves away. Complete overlap of the emission line with the absorption line ${ }^{*}$ will take place when

$$
\mathrm{E}_{\mathrm{a}}=\mathrm{E}_{\gamma}=\mathrm{E}_{\mathrm{e}}\left[1+\frac{\mathrm{v}_{\mathrm{r}}}{\mathrm{c}}\right],
$$

where $v_{r}$ is the velocity for which complete overlap, or maximum absorption takes place. For velocities other than $v_{r}$ only partial overlap of the emission and absroption lines will take place, resulting in a decreased amount of absorption.

The shape of the composit curve (the intensity of the radiation transmitted through the absorber plotted against the source velocity) will depend on the shape of the emission and absorption lines. If. both lines are natural then the composit curve will be nearly

As in the case of emission, the absorption spectrum (the number of gamma rays resonantly absorbed per unit.energy range) will be Lorentzian in shape with natural width. 
Lorentzian with a width of twice natural ${ }^{20} ; 0.20 \mathrm{~mm} / \mathrm{sec}$ for $\mathrm{Fe}^{57}$. The usual convention used in quoting line widths is to quote the width of the composit curve (natural width is $0.20 \mathrm{~mm} / \mathrm{sec}$ using this convention); this convention is used here. It should also be noted that the composit curve is commonly referred to as the "line shape", this practice is also followed here.

The resolution of the line shape measurements is limited by the width of the absorption line. If the absorption line is broader than natural, either because of unresolved structure of the Iine or thickness broadening ${ }^{20}$, the resolution of the measurement is reduced. Therefore, a natural line width absorber has been used; a $7.6 \mathrm{mg} / \mathrm{cm}^{2}$ iron foil containing a natural abundance $(2 \%)$ of $\mathrm{Fe}^{57}$. Due to the nuclear Zeeman effect the absorber has six absorption lines. Of the six Iines only the one closest to zero velocity has been used; the position of this line is $0.835 \mathrm{~mm} / \mathrm{sec}$ from the centroid of the six line pattern.

Two different drives are used to provide the relative motion between source and absorber. One of these is the speaker drive described by Nees ${ }^{22}$. The other is a constant velocity mechanical drive which is based on a similar drive described by Nussbaum et. al. 23 Both drives are capable of measuring natural width lines. The speaker drive is faster and is useful when the position and structure of the emission line is unknown. The mechanical drive has the advantage of greater velocity resolution; it is useful once the position of the line has been determined with the speaker drive. 


\section{SOURCE PREPARATION}

In order to prepare Mössbauer sources the active atom, in this case $\mathrm{Fe}^{57}$, must be substituted for one of the host atoms. The procedure used here is a two step process. First, the activity, in the form of $\mathrm{Co}^{57}$, is deposited onto the surface of the host metal. Second, the source is placed in a furnace and the activity diffused into the bulk of the host metal.

\section{Electrolytic Deposition}

The process used here to deposit the activity onto the surface of the host metal was that of electrolytic deposition. The central problem of this technique is to deposit microgram quantities of $\mathrm{Co}^{57}$ onto the surface of the host; therefore, macroscopic platting techniques cannot be employed. The technique of electrolytic deposition has been throughly investigated elsewhere $24-26$.

The electrolyte used is the same as that used by Dèzsi ${ }^{25}$ since the reported deposition rate was greater than that used by stephen ${ }^{24}$. The composition of the electrolyte is an aqueous solution of $2.5 \mathrm{~g} / 100 \mathrm{ml}$ of hydrazine hydrate and $2.5 \mathrm{~g} / 100 \mathrm{ml}$ ammonium dicitrate; the $\mathrm{pH}$ of the electrolyte is adjusted to 10 with ammonium hydroxide. The electrolytic cell in which the deposition is carried out is shown in figure 2. The $250 \mathrm{ml}$ beaker provides a closed atmosphere for the cell; the top is sealed with Saran wrap while the cell is in operation. The pH of the electrolyte can be maintained at 10 for about six hours without the addition of more ammonium hydroxide. Electrical contact between the platform and the motor shaft is made through ball 


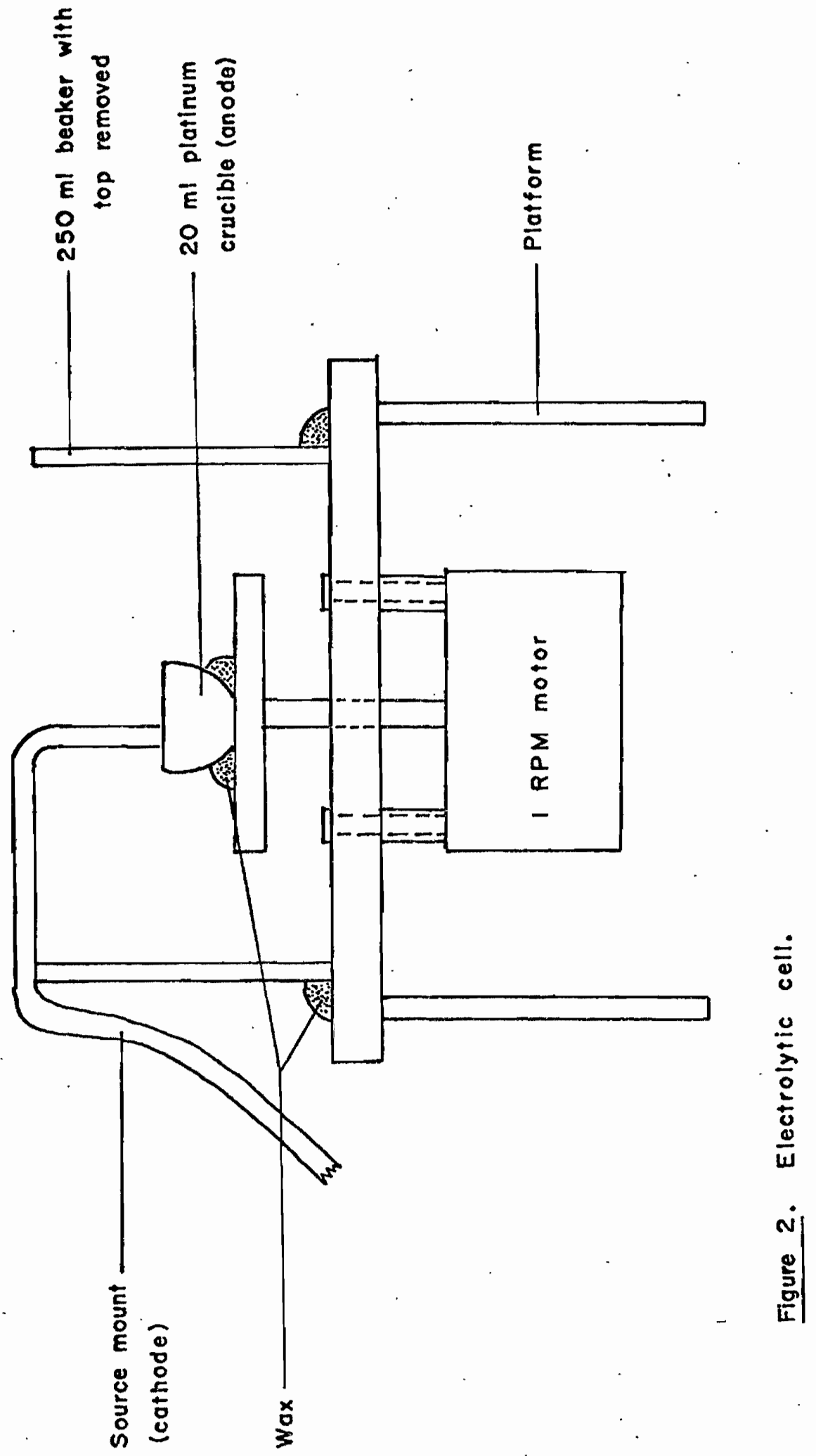


bearings. After the cell had been in use for a while the corrosive atmosphere of ammonia tended to form a nonconductive layer on the ball bearings. The effect of this nonconductive layer is to cause an intermittent current flow through the cell. It is believed that this Intermittent current flow has an adverse effect on the deposition process.

The purpose of the source mount is to hold the host metal in the electrolyte and to provide for an electrical connection between the host and the power supply. The host is typically cylindrical in shape; the height of the cylinder is about $0.5 \mathrm{~mm}$. The thickness of the host metal makes it undesireable to deposit activity onto the back or side of the host since the $14.4 \mathrm{keV}$ gamma ray originating from the back or side will be strongly attenuated by mass absorption. This attenuation will increase the relative intensity of the high energy gamma rays ( 122 and $136 \mathrm{keV}$ ), causing an increase in the background intensity. Therefore, the source mount must also insulate the back and side of the host from the electrolyte.

The source mount used is shown in figure 3. The purpose of the paper tape is to fasten the copper wire to the host metal until the polystyrene dries. Trichloroethylene is a solvent for both the polystyrene and the adhesive on the paper tape. It was found that when thick coats of plastic were applied to the source mount the paper tape was loosened from the back of the host before the plastic finished drying. This results in a poor electrical connection to the host. Therefore, the plastic should be applied only in thin layers. The plastic is not completely resistant to chemical attack. After the 
SIDE VIEW

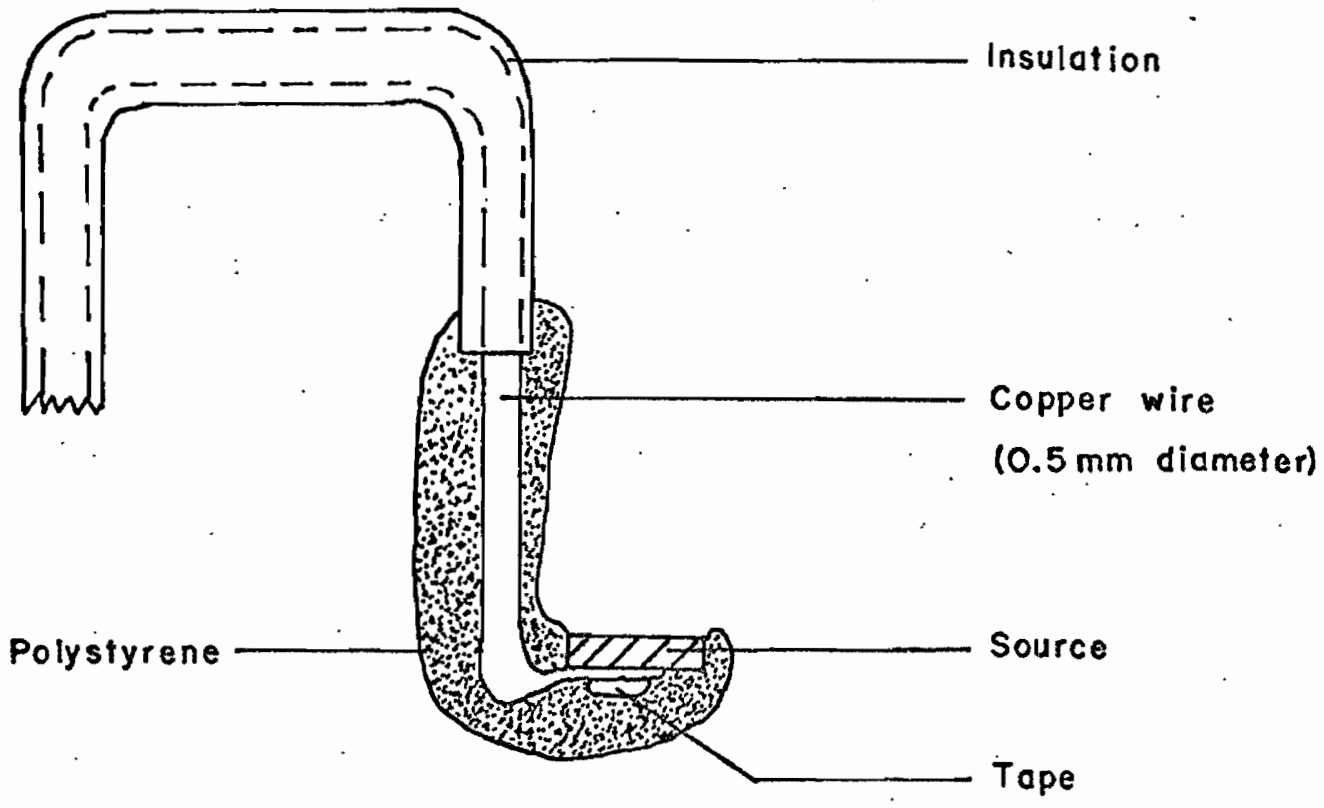

BOTTOM VIEW

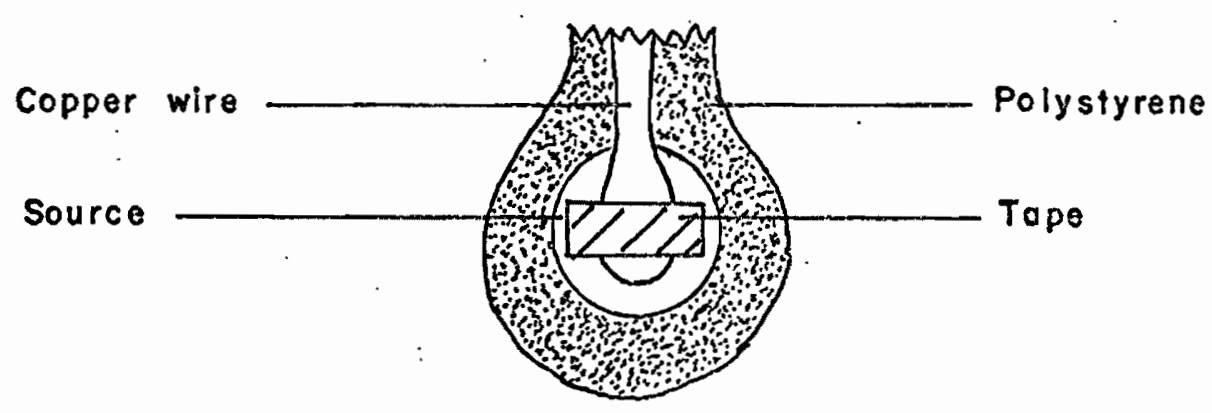

Figure 3. Source mount: 
plastic has been emersed in the electrolyte for more than 48 hours it begins to soften. However, this has not as yet been a problem since the deposition times have been shorter than 48 hours. There are two advantages that the polystyrene has over other materials which have been tried as insulators: Very little activity adheres to the plastic and it is easily removed after the deposition has been completed. To remove the plastic a razor blade is inserted between the back of the host and the copper wire; the plastic is then easily peeled away from the host.

After the host is mounted, the top surface is degreased with trichloroethylene and then lightly etched since the plated metal adheres poorly to a smooth surface ${ }^{25}$. In the case of copper the etch was a concentrated potassium dichromate solution. For gold the etch was composed of equal parts of a $10 \%$ aqueous solution of $\mathrm{KCN}$ and a saturated aqueous solution of $\mathrm{K}_{2} \mathrm{~S}_{2} \mathrm{O}_{8}$.

The procedure used in the deposition is as follows: The platinum crucible was filled with about $3 \mathrm{ml}$ of electrolyte and the $\mathrm{Co}^{57}$ in the form of $\mathrm{CoCl}_{2}$ in $0.1 \mathrm{~N} \mathrm{HCl}$ was added; the source was then placed in the cell, the current turned on, and the deposition carried out. The constant current power supply used is similar to that used by Stephen ${ }^{24}$. Every eight hours a drop of hydrazine hydrate was added to the cell. About every six hours the pH of the electrolyte was readjusted to 10 with ammonium hydroxide. Occasionally it was necessary to carry out the deposition overnight. When this happened the electrolyte was adjusted to a $\mathrm{pH}$ of 11 before leaving for the night; in the morning the $\mathrm{pH}$ was usually found to be about 9 . 
The progress of the deposition process was monitored in the following manner: At regular intervals $0.035 \mathrm{ml}$ samples of the electrolyte were removed from the cell; the samples were placed under a NaI scintillation counter and the countrate of the $122 \mathrm{keV}$ gamma ray was measured. The results of the deposition onto copper and gold are shown in figure 4. The error bars do not include the volumetric sampling error which is estimated to contribute another $5 \%$.

\section{Copper}

After electrodeposition and prior to diffusion the surface of the host is always black ${ }^{27}$; after diffusion the black color disappears. The plated layer of $\mathrm{Co}^{57}$ is not thich enough to cause this black color, therefore, something else besides $\mathrm{Co}^{57}$ is being deposited onto the surface of the host. At present it is not known what this substance is. The absence of the black color after diffusion admits the possibility that this foreign substance is diffused into the bulk of the host along with the $\mathrm{Co}^{57}$. Therefore, a copper source was made in order to determine if these foreign atoms interfere with the $\mathrm{Co}^{57}$ atoms. Copper was chosen for two reasons:

1) The presence of the foreign atoms may result in a quadrupole interaction or a number of different. chemical states for the $\mathrm{Co}^{57}$ atoms. This in turn will result in a broad line. Previously ${ }^{15}$ we have been able to prepare copper sources having natural width lines. Therefore, any line broadening in a copper source prepared using the technique of electrolytic deposition would clearly indicate that the foreign atoms interfere 


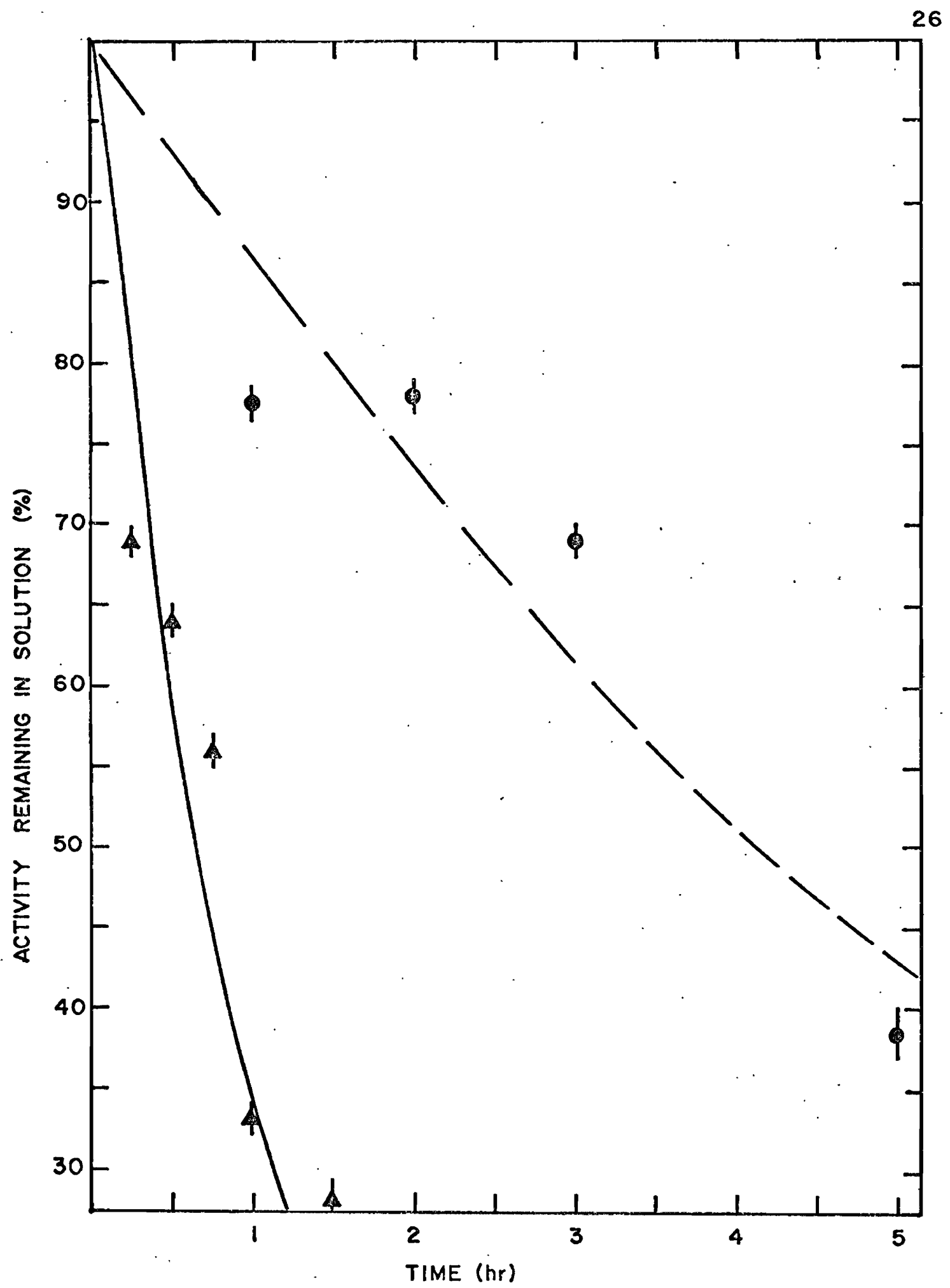

Figure 4. Results of the electrodeposition of $\mathrm{Co}^{57}$ onto copper (A) and gold (O). The solid curve is the result that Dèzsl obtained for deposition onto copper. 
with the $\mathrm{Co}^{57}$ atoms.

2) The presence of the foreign atoms may also effect the mean-squared displacement, and hence the Debye-Waller factor, of the $\mathrm{Co}^{57}$ atoms. The $f$ of $\mathrm{Fe}^{57}$ in copper is well known ${ }^{15}$ and can therefore be used as a standard against which to compare the f obtained from a copper source prepared by electrolytic deposition.

The piece of copper used was $99.995 \%$ pure. It was cut with a jewelers saw from a single crystal obtained from Semi-Metals Inc. The deposition was carried out for two hours at a current density of $100 \mathrm{~mA} / \mathrm{cm}^{2}$. The deposition rate was in agreement with that obtained by Dèzsi ${ }^{25}$ as shown in figure 4. The source, Cu III, was diffused in an atmosphere of hydrogen for ten minutes at $1000^{\circ} \mathrm{C}$. After diffusion, the surface of the source was cleaned with pumice soap and water. The Iine shape of the source was measured and found to have natural width. The room temperature $f$ was measured and found to be in agreement with the previously obtained value ${ }^{15}$. Therefore, contrary to the results that Qaim 27 obtains, it appears that useable sources having natural width lines can be prepared using the technique of electrolytic deposition.

Diffusion of Gold

Diffusion of the gold sources was carried out in an atmosphere of hydrogen. Hydrogen was chosen because it will prevent any oxygen which is present from interfering by forming oxides. The source, inside a vycor tube, was placed in a hot furnace, kept there for the desired length of time, and then removed and quenched by putting the vycor tube 
Into a beaker of cold water. For the short diffusion times used in preparing the gold sources the heating rate becomes important. Therefore, the heating curve from room temperature to $510^{\circ} \mathrm{C}$ is shown in figure 5. This curve was obtained by attaching a chromel-alumel thermocouple to a piece of gold placed inside the vycor tube. The vycor tube was then placed in the hot furnace.

The quenching rate is too rapid to measure using the thermocouple. The only estimate of the quenching rate we have is that when quenched from $1000^{\circ} \mathrm{C}$, the source stops glowing (indicating a temperature less than $700^{\circ} \mathrm{C}$ ) after it has been out of the furnace, and in the beaker of water, for about ten seconds. Therefore, the initial quenching rate is greater than $30^{\circ} /$ sec.

GoId

Three gold sources were made, but measurements were made on only two of them. All three sources were cut from a $99.995 \%$ pure single crystal obtained from Semi-Metals Inc. The first source to be made was Au II. The deposition was carried out for two hours at a current density of $40 \mathrm{~mA} / \mathrm{cm}^{2}$ and for another 18 hours at a current density of $80 \mathrm{~mA} / \mathrm{cm}^{2}$. The rate of deposition is shown in figure 4. Au II was diffused at the same time as $\mathrm{Cu}$ III (ten minutes at $1000^{\circ} \mathrm{C}$ ).

Using a Xe-CH 4 filled proportional counter and an FET preamp, the Au $I_{r} x$-ray is unresolved from the nonresonant $\mathrm{Fe}^{57} 14.4 \mathrm{keV}$ gamma ray, (see figure 6). A correction (discussed in part III below) must therefore be applied to the measured $f$ in order to obtain absolute values of the Debye-Waller factor. As the intensity of the gold I $\mathrm{x}$-ray is increased relative to the intensity of the $14.4 \mathrm{keV}$ gamma ray, 


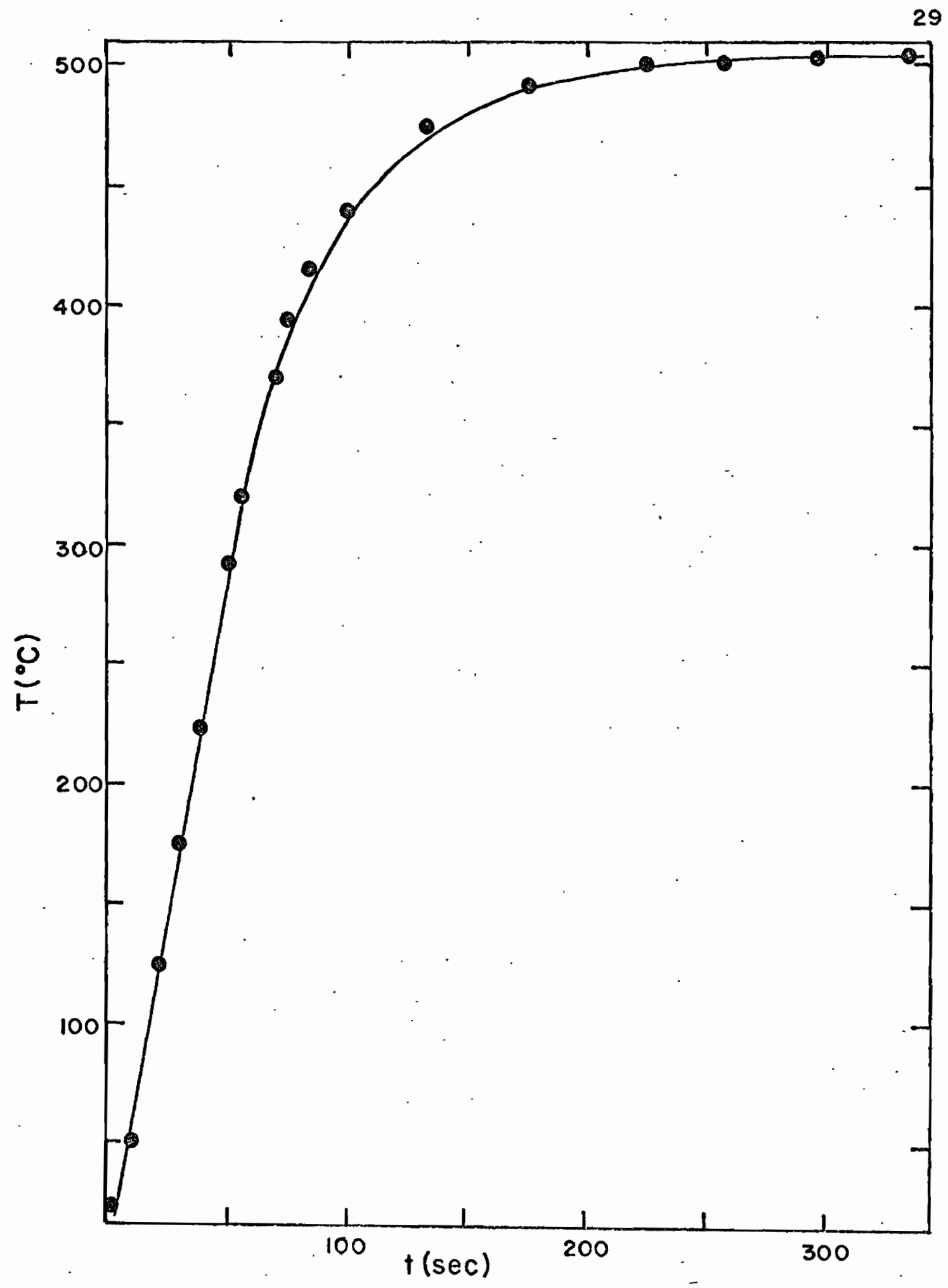

Figare 5. Heating rate of the surface of a Au somple placed inside the Vycor fube. The furnace temperature was $512{ }^{\circ} \mathrm{C}$. 


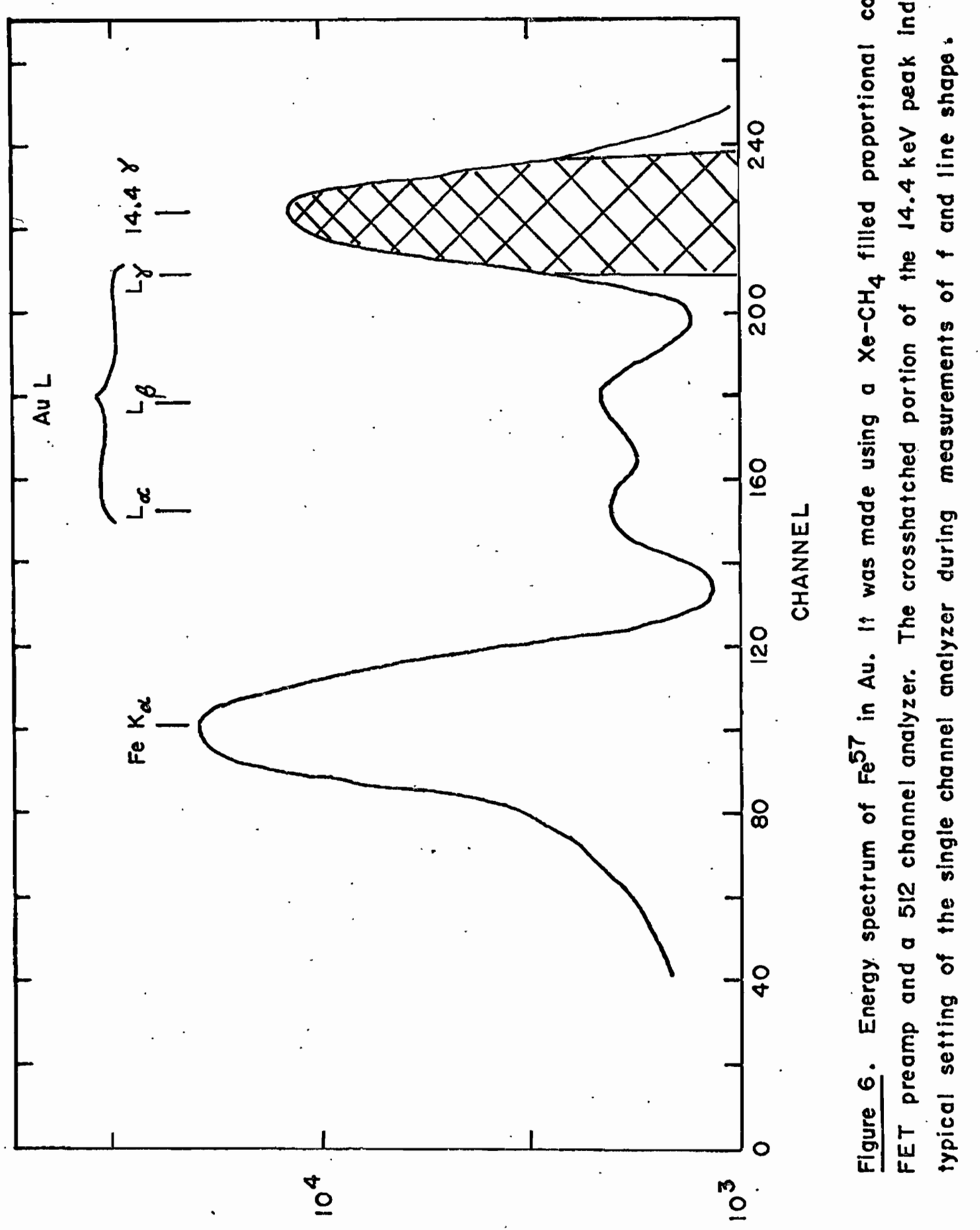

SLNกOS 
the magnitude of the $x$-ray correction will increase. For Au II, due to deep diffusion, the ratio $I_{x-r a y} / I_{14.4}$ was found to be slightly greater than unity, (the intensity of the gold $I_{B} x$-ray was used for $I_{x-r a y}$ ). It was felt that the corresponding $x$-ray correction would be too large to warrant the use of $\mathrm{Au}$ II in making absolute $f$ measurements.

Au III was made next. It was plated for 17 hours at a current density of $40 \mathrm{~mA} / \mathrm{cm}^{2}$ and then for another two hours at a current density of $80 \mathrm{~mA} / \mathrm{cm}^{2}$. The amount of activity deposited onto the surface of the source was $0.85 \mathrm{mCi}$. Because of the results of the diffusion of Au II, it was decided to start the diffusion process at $500^{\circ} \mathrm{C}$ for two minutes. There were two criteria which were used to determine when the activity was diffused deeply enough into the bulk of the source:

1) Iine shape measurements - When the activity is initialIy deposited onto the surface of the host the line width will be broad due to the large number of surface sites which the $\mathrm{Fe}^{57}$ atoms can occupy. As the activity is diffused into the bulk of the source', the line characteristic of the bulk site will be enhanced at the expense of the surface states. Therefore, by measuring the width of the line after each diffusion it is possible to determine when most of the activity is in the bulk site.

2) $\mathrm{X}$-ray ratio - As previously discussed, the ratio $I_{x-\text { ray }} / I_{14.4}$ gives an indication of the magnitude of the $x$-ray correction. After our experience with Au II 
It was decided that in order for a source to be useable the $x$-ray ratio must be less than unity.

A total of seven diffusions of Au III were made; these are summarized in table I.

After the seventh diffusion it was decided that the $x$-ray ratio was too close to unity to try another diffusion. The surface of the source was etched for $1 \frac{1}{2}$ minutes in aqua regia to remove any undiffused activity. Neither drive was producing reliable line shapes at this time. However, subsequent line shape measurements on Au IV and Au IIIB (see below) lead us to believe that the line width was $0.24 \pm 0.01$ $\mathrm{mm} / \mathrm{sec}$. After the high temperature $f$ measurements, Au III was rediffused for three minutes at $850^{\circ} \mathrm{C}$; this source has been labled Au IIIB. The third source to be made was $A$ U IV. Au IV was plated for $5 \frac{1}{2}$ hours at a current density of $120 \mathrm{~mA} / \mathrm{cm}^{2}$ and then for another 12 hours at a current density of $60 \mathrm{~mA} / \mathrm{cm}^{2}$. The amount of activity deposited onto the surface of the source was $1.2 \mathrm{mCi}$. Au IV was diffused for three minutes at $750^{\circ} \mathrm{C}$. The surface was then etched for one minute with aqua regia. The line width of the source was broad so it was rediffused for two minutes at $1000^{\circ} \mathrm{C}$. After another two minute etch in aqua regia the line width was found to be $0.235 \pm 0.005 \mathrm{~mm} / \mathrm{sec}$, (see figure 7 ).

\section{X-RAY CORRECTION}

The high energy radiation (122 and $136 \mathrm{keV}$ gamma rays) which originates within the bulk of the host will produce $\mathrm{x}$-rays characteristic of the host. In order to make $f$ measurements the radiation 
TABLE I

DIFFUSION PROCESS USED TO MAKE Au III

\begin{tabular}{|c|c|c|}
\hline$T\left({ }^{\circ} \mathrm{C}\right)$ & $t(\min )$ & $I_{x-r a y} / I_{14.4}$ \\
\hline 22 & - & $1 / 4.4$ \\
515 & 2 & $1 / 4.2$ \\
515 & 4 & $1 / 4.0$ \\
600 & 4 & $1 / 4.3$ \\
600 & 10 & $1 / 3.8$ \\
650 & 10 & $1 / 3.8$ \\
650 & 10 & $1 / 3.2$ \\
700 & 4 & $1 / 2.2$ \\
\hline
\end{tabular}




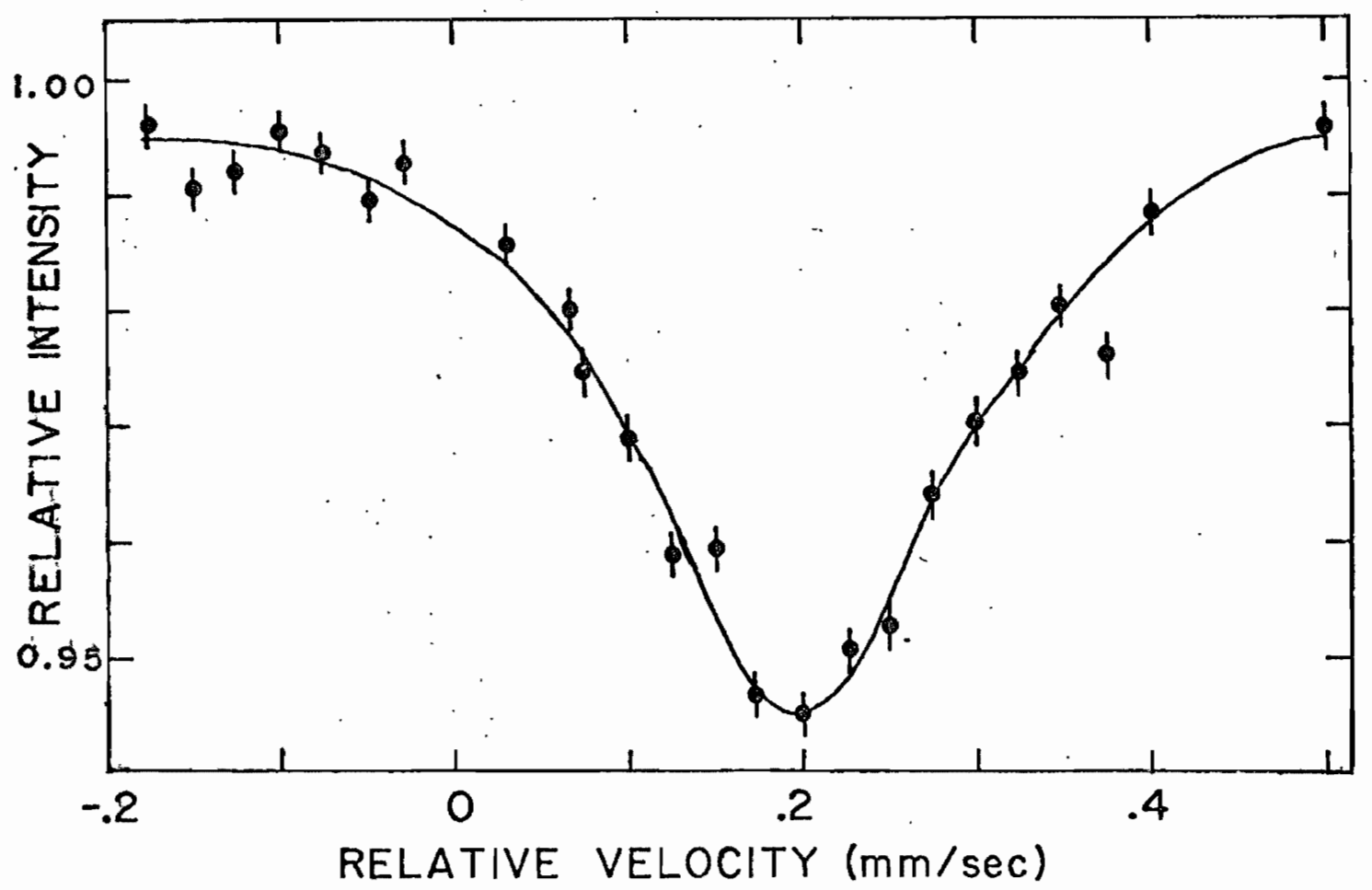

Figure 7. Line shape of AuIV made using the mechanical drive and one of the inner lines of an iron absorber. This measurement was made after the second diffusion and after the surface had been etched. The solid line is a Lorentzian curve centered about $0.20 \mathrm{~mm} / \mathrm{sec}$ with a full width of half maximum of $0.235 \mathrm{~mm} / \mathrm{sec}$. 
detection system must distinguish between the $14.4 \mathrm{keV}$ gamma ray and the host $x$-rays, (see figure 6 ). This separation is accomplished by using a single channel analyzer ( $S C A$ ); a typical window setting for the SCA is shown in figure 6. Since the gold L/ $\gamma x$-ray is unresolved from the $14.4 \mathrm{keV}$ gamma ray the SCA will not completely exclude the $\mathrm{I}_{\gamma}$ $x$-ray from the $14.4 \mathrm{keV}$ window. The countrate through the window will therefore be increased due to the presence of the $x$-ray and a correction must be applied to the measured $f$ values in order to obtain absolute values for the Debye-Waller factor.

The method developed to correct for the $I_{\gamma} x$-ray makes use of the $\mathrm{K}$ absorption edge of $\mathrm{Br}$; this method was suggested by Housley et. al. 28 The $B r K$ edge $(13.475 \mathrm{keV})$ lies betwee the $L_{\gamma_{1}}$ gold $x$-ray (this $\dot{x}$ - ray contributes $53 \%$ to the total intensity of the gold $I_{Y} x-r a y$, see table III) and the $14.4 \mathrm{keV}$ gamma ray. Because of the position of the $\mathrm{K}$ edge, the $14.4 \mathrm{keV}$ gamma ray is more strongly attenuated by a $\mathrm{NaBr}$ absorber than is the $I_{\gamma} x$-ray.

To determine the value of the Debye-Waller factor corrected for the presence of the gold $I_{\gamma} x$-ray, four measurements were made using a wide, black absorber ${ }^{*}$ and a nonresonant absorber (either NaBr or a $\mathrm{Cu}$ foil). The experimental setup is shown in figure 8. The first measurement, $I_{\infty}$, is the intensity transmitted through the wide, black absorber with the source driven by the speaker at $60 \mathrm{~Hz}$; the maximum velocity obtained by the source was about $10 \mathrm{~cm} / \mathrm{sec}$. The measured

For a discussion of the properties of the black absorber the reader is referred to reference 28 : 


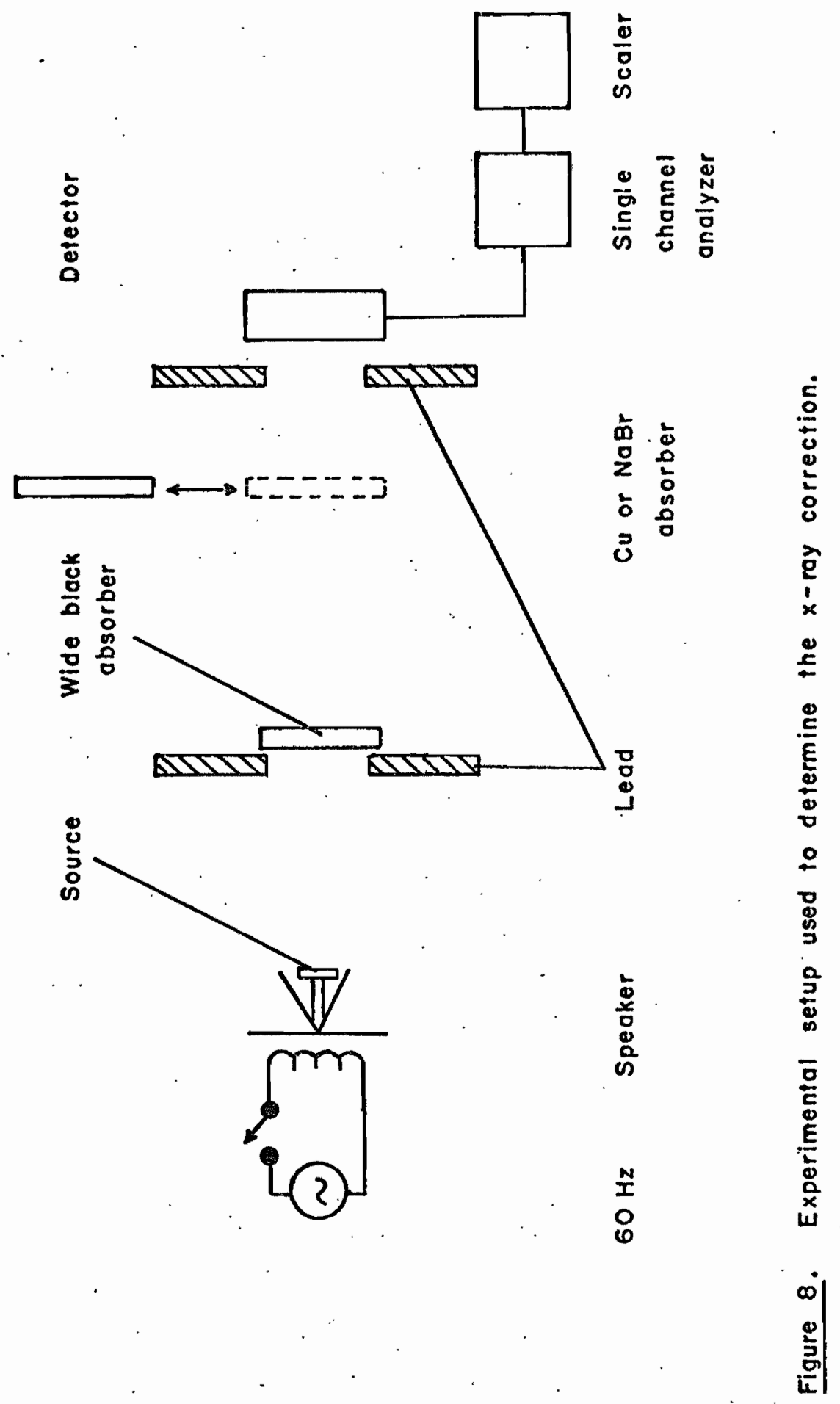


intensity is given by

$$
I_{\infty}=I_{R}+I_{N R}+I_{B}
$$

where $I_{R}$ is the resonant or zero phonon fraction of the $14.4 \mathrm{keV}$ radiation, $I_{N R}$ is the nonresonant fraction, $I_{X}$ is the intensity of the Ir $x$-ray contributing to the $14.4 \mathrm{keV}$ window, and $I_{B}$ is the background intensity due to the higher energy radiation. The second measurement, $I_{\infty}^{\prime}$, is the same as $I_{\infty}$ except that one of the nonresonant absorbers is placed between the detector and the black absorber. The measured intensity is

$$
I_{\infty}^{\prime}=\alpha\left(I_{R}+I_{N R}\right)+\xi I_{X}+\delta I_{B}
$$

where $\alpha, \xi$, and $\delta$ are the transmission ratios (the ratio of the transmitied intensity to the incident intensity) of the $14.4 \mathrm{keV}$ gamma ray, the $L_{\gamma} x$-ray, and the background radiation respectively. It is assumed here that there is only one $x$-ray which contributes to $I_{X}$, or (as is the case with the $\mathrm{Cu}$ foil absorber) that the transmission ratio is the same for all the $x$-rays. The third measurement, $I_{0}$, is made with the source at rest with respect to the resonant wide black absorber and the nonresonant absorber removed. The transmitted intensity is

$$
I_{0}=I_{N R}+I_{X}+I_{B}
$$

The last measurement, $I_{0}^{\prime}$, is the same as $I_{0}$ except that the nonresonant absorber is added. The measured intensity is

$$
I_{0}^{\prime}=\alpha I_{N R}+\xi I_{X}+\delta I_{B}
$$

From these measurements we obtain $f^{\prime}$ defined by 


$$
\begin{aligned}
P^{\prime} & =1-\frac{I_{0}-I_{0}^{\prime}}{I_{\infty}-I_{\infty}^{\prime}} \\
& =\frac{(1-\alpha) I_{R}}{(1-\xi) I_{X}+(1-\delta) I_{B}+(1-\alpha)\left(I_{R}+I_{N R}\right)} \\
& =\frac{\frac{I_{R}}{I_{R}+I_{N R}}}{1+\frac{(1-\xi) I_{X}+(1-\delta) I_{B}}{(1-\alpha)\left(I_{R}+I_{N R}\right)}}
\end{aligned}
$$

The corrected Debye-Waller factor $f_{0}$ is given by

$$
f_{0}=\frac{I_{R}}{I_{R}+I_{N R}}
$$

Equation (43) can now be written

$$
f_{0}=\dot{f}^{\prime}\left[1+\frac{(1-\xi) I_{X}+(1-\delta) I_{B}}{(1-\alpha)\left(I_{R}+I_{N R}\right)}\right]
$$

As previously stated, it has been assumed that there is only one $x$-ray contributing to $I_{X}$. When there is more than one $x$-ray equation (45) becomes

$$
f_{0}=f^{\prime}\left[1+\frac{I_{X} \sum_{n}\left(1-\xi_{n}\right) \psi_{n}+(1-\delta) I_{B}}{(1-\alpha)\left(I_{R}+I_{N R}\right)}\right],
$$

where $\xi_{n}$ is the transmission coefficient of the $n^{\text {th }} x$-ray and $I_{X} \psi_{n}$ gives the contribution of the $n^{\text {th }} x$-ray to the total $x$-ray intensity.

The NaBr absorber was made by soaking a filter paper in a $50 \%$ saturated aqueous $\mathrm{NaBr}$ solution; the filter paper was then dried under a heat lamp. An energy spectrum of Au III was taken through the absorber and compared to one taken for the same length of time without the absorber in place. From this comparison the transmission ratio of 
the $14.4 \mathrm{keV}$ radiation was determined. It was also determined that there was essentially no attenuation of the background radiation. From the calculations of Storm and Isreal ${ }^{29}$, the mass absorption coefficient for the $14.4 \mathrm{keV}$ radiation was determined to be $127 \mathrm{~cm}^{2} / \mathrm{g}$, (see figure 9): Using the absorption law for gamma rays

$$
\left(I / I_{0}\right)=\exp (-\mu x)
$$

and the transmission coefficient for the $14.4 \mathrm{keV}$ radiation, the thickness of the absorber can be determined

$$
x=-\frac{\ln (\alpha)}{127 \mathrm{~cm}^{2} / \mathrm{g}}
$$

Jsing the absorber thickness and the mass absorption coefficients for the gold $I_{\gamma} x$-rays, the transmission ratios for the $x$-rays can be determined

$$
\xi_{n}=\exp \left(-\mu_{n} x\right)
$$

The parameters for this absorber are given in tables II and III. In table III only those $x$-rays which contribute more than $1 \%$ to the total $\mathrm{x}$-ray intensity are considered. Using these results, equation (46) can be written

$$
f_{0}=f_{\mathrm{NaBr}}^{\prime}\left[1+\frac{0.59 I_{X}}{I_{R}+I_{N R}}\right]
$$

The parameters for the $\mathrm{Cu}$ foil are aiso given in table II. For the copper foil equation (46) can be written

$$
f_{0}=f_{C u}^{\prime}\left[1+\frac{I_{X}}{I_{R}+I_{N R}}\right]
$$

Dividing equation (50) by (51) and defining the quantity $\Delta=$ $I_{X} /\left(I_{R}+I_{N R}\right)$ gives 


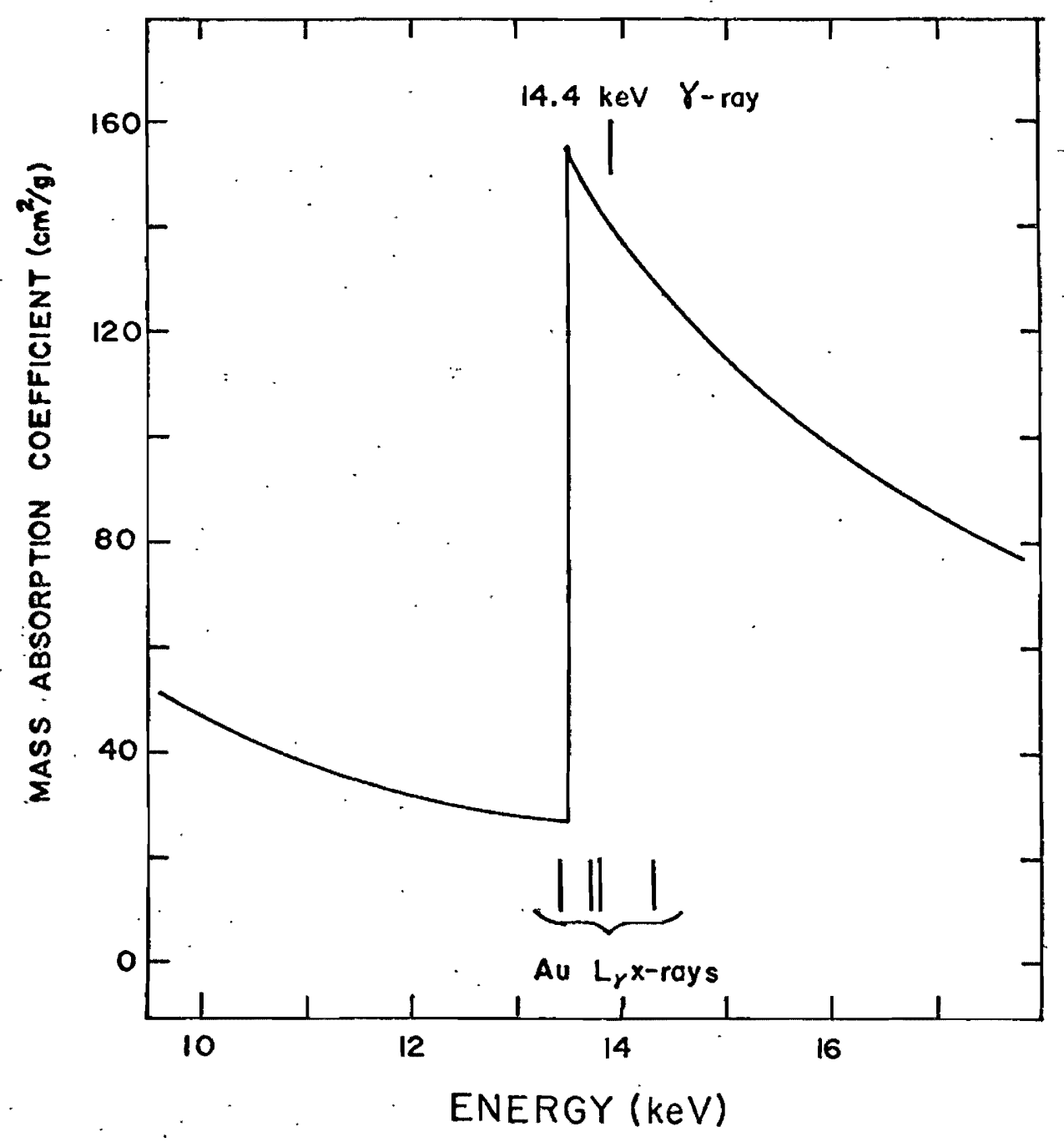

Figure 9. K absorption edge of $\mathrm{Br}$. 
41

TABLE II

ABSORBER PARAMETERS

\begin{tabular}{|c|c|c|c|c|}
\hline Absorber & $x\left(\mathrm{mg} / \mathrm{cm}^{2}\right)$ & $\alpha$ & $\delta$ & $\xi$ \\
\hline $\mathrm{NaBr}$ & 5.0 & 0.52 & 1 & $(a)$ \\
$\mathrm{Cu}$ & 66. & 0.004 & 0.98 & 0.004 \\
\hline
\end{tabular}

(a) see table III. 
TABLE III

TRANSMISSION COEFFICIENTS AND NORMALIZED

INTENSITIES FOR THE AU

$$
{ }^{I} \gamma \mathrm{X} \text {-RAYS }
$$

\begin{tabular}{|c|c|c|c|c|c|}
\hline Designation & $\begin{array}{c}\text { Energy } \\
(\mathrm{keV})\end{array}$ & $\begin{array}{c}\text { Relative } \\
\text { Intensity }\end{array}$ & $\begin{array}{c}\text { Normalized } \\
\text { Intensity } \\
\psi_{\mathrm{n}}\end{array}$ & $\begin{array}{c}\mu^{\mathrm{n}}{ }^{\mathrm{b}} \\
\left(\mathrm{cm}^{2} / \mathrm{g}\right)\end{array}$ & ${ }_{\mathrm{n}}$ \\
\hline 1 & 13.38 & 26.2 & 0.53 & 21 & 0.9 \\
2 & 13.71 & 7.8 & 0.16 & 145 & 0.5 \\
3 & 13.81 & 9.6 & 0.19 & 142 & 0.5 \\
6 & 13.73 & 2.9 & 0.06 & 144 & 0.5 \\
$4 / 1$ & 14.28 & 1.4 & 0.03 & 130 & 0.5 \\
$4 / 2$ & 14.30 & 1.7 & 0.03 & 130 & 0.5 \\
\hline
\end{tabular}

(a) These values were taken from reference 29.

(b) These values were taken from figure 9. 


$$
\frac{f_{\mathrm{NaBr}}^{\prime}}{f_{\mathrm{Cu}}^{\prime}}=\frac{1+\Delta}{1+0.59 \Delta}
$$

or

$$
\Delta=\frac{f_{\mathrm{NaBr}}^{\prime}-f_{\mathrm{Cu}}^{\prime}}{f_{\mathrm{Cu}}^{\prime}-0.59 f_{\mathrm{NaBr}}^{\prime}}
$$

Defining $c_{X}=1 /(1+\Delta)$ and using equation (51), the corrected DebyeWaller factor is given by

$$
f_{0}=\frac{f_{C u}^{\prime}}{C_{x}}
$$

Since the $x$-ray correction will depend on the setting of the single channel analyzer, it will change each time this setting is changed. Instead of again using the $\mathrm{NaBr}$ absorber to determine the new correction the following method was used; the corrected value of the Debye-Waller factor for Au has already been determined, therefore, the new correction can be found using equation (54) and measuring the new value of $\mathrm{f}_{\mathrm{Cu}}^{\prime}$.

The uncertainty in the determination of $\Delta$ will be quite large since $\left(f_{\mathrm{NaBr}}^{\prime}-f_{\mathrm{Cu}}^{\prime}\right)$ is small. The uncertainty in the values obtained for $f_{\mathrm{NaBr}}^{\prime}$ and $f_{\mathrm{Cu}}^{\prime}$ was $0.5 \%$. This leads to a $20 \%$ uncertainty in $\Delta$ for the sources used here. However, the uncertainty in the value of the $x$-ray correction $C_{X}$ is much better than that for $\Delta$. A $20 \%$ uncertainty in $\Delta$ leads to an uncertainty of $0.6 \%$ in the determination of the $x$-ray correction. There is one more factor which can strongly effect the accuracy of the $x$-ray correction; i.e. the assignment of the relative $x$-ray intensities used in table III. These intensities are based on theoretical calculations; the uncertainty of these calculations is 
estimated to be about $0.5 \%{ }^{30}$. The ratio of the $K_{\alpha_{1}}$ to the $K_{\alpha_{2}} x$-ray has been experimentally measured for a number of elements with $2>50^{31}$. The results of these measurements are in good agreement with the calculated intensities.

\section{IV. $f$ MEASUREMENTS}

The furnace used to make the $f$ measurements is shown in figure 10. The aluminum ring used to hold the mica in place limits the maximum temperature which can be obtained. Also, the cooling of the brass shell is insufficient to allow the temperature of the heated shield to be much greater than about $500^{\circ} \mathrm{C}$. The platinum resistor is used as a temperature sensor for a commercial controller. This combination will maintain the temperature of the source constant to within $\pm \frac{1}{2} \mathrm{~K}$ below about $700 \mathrm{~K}$.

A black conductive layer forms on the surface of the alundum feedthroughs. It is not known whether this is from the binder in the Saureisen or is intrinsic in the alundum. Above $700 \mathrm{~K}$ the Saureisen becomes quite conductive. The combination of these two effects acts to prevent the temperature controller. from working properly. Therefore, above $700 \mathrm{~K}$ the regulation of the source temperature is carried out by hand; the uncertainty of the source temperature under these conditions is about $\pm 5^{\circ}$.

The measurements have been carried out in a vacuum which was better than $10^{-4}$ Torr. The black, wide absorber technique ${ }^{28}$ has been used to make the $f$ measurements. Besides the x-ray correction discussed above, the measured $f$ values must also be corrected for re-radi- 


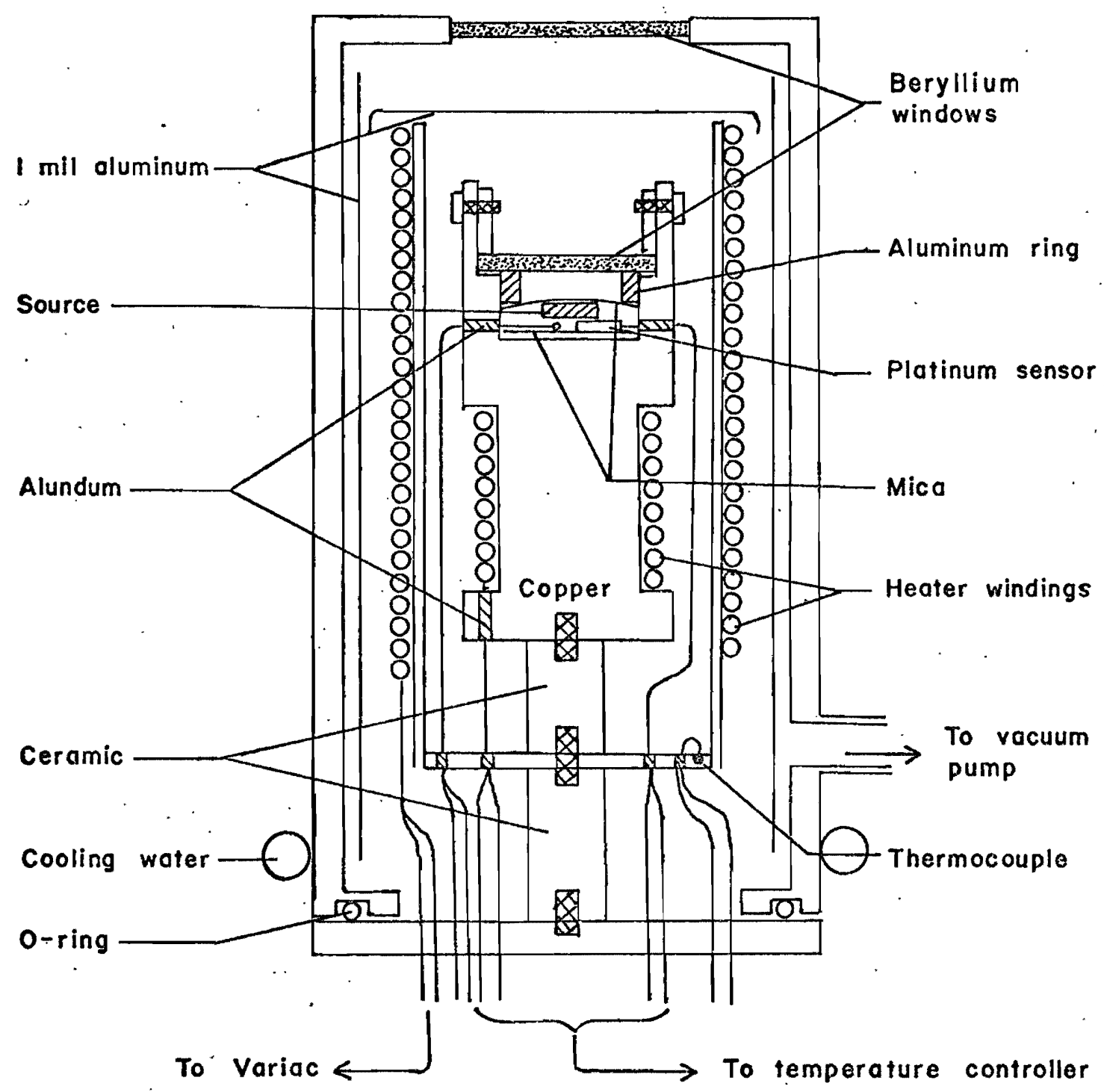

Figure 10. Axially symmetric furnace used for the high femperature $f$ measurements. Scureisen is used to insulate the thermocouple wires and for potfing in the heater windings. 
ation from the black absorber, blackness of the black absorber, finite amount of resonant absorption at $I_{\infty}$, and Compton scattering from the beryigum windows used in the construction of the furnace. These corrections have been discussed by Housley ${ }^{32}$ and Nees ${ }^{22}$.

The statistical uncertainty of the measurements of the DebyeWalier factor is less than $0.5 \%$. There is a $0.6 \%$ uncertainty in the determination of the $x$-ray correction and a $0.5 \%$ uncertainty in the determination of the blackness correction for the wide black absorber (see reference 15). It is estimated that these two errors combine to Eive $0.8 \%$ uncertainty in the absolute accuracy of the measurements. Therefore, the total uncertainty of the measurements, statistical plus ebsolute, is estimated to be $1.3 \%$. 
DATA PRESENTATION AND INTERPRETATION

The measured temperature dependence of the exponent of the DebyeWaller factor of $\mathrm{Au}$ III, between room temperature and $760 \mathrm{~K}$, and $\mathrm{AU} \mathrm{IV}$, between room temperature and $829 \mathrm{~K}$, is shown in figure 11. The expected temperature dependence of $(-\ln f)=2 W$ is given by equation (23). The solid lines in figure 11 have been calculated from equation (23) using the Debye model, equation (9), for $(2 W)_{H}$; the method used to fit the two parameters $\varepsilon(-2)$ and $\theta_{D}(-2)$ to the data ${ }^{*}$ is described in reference 15.

Above $700 \mathrm{~K}$ the Debye-Waller factor of both $\mathrm{Au}$ III and $\mathrm{Au}$ IV is greater than the value predicted by equation (23). Since the DebyeWaller factor is related to the mean squared displacement of the $\mathrm{Fe}^{57}$ Impurity atom through equation (22), this deviation means that the average mean squared displacement of the $\mathrm{Fe}^{57}$ atoms is smaller than expected. Furthermore, at two temperatures, $765 \mathrm{~K}$ and $829 \mathrm{~K}$, the $f$ of Au IV was observed to increase with time, (see figure 12). This behavior can occur only if the $\mathrm{Co}^{57}$ (and $\mathrm{Fe}^{57}$ ) atoms are diffusing from one lattice site (referred to below as site A) to another, nonequivalent

*For reasons which will become apparent, only the data below $700 \mathrm{~K}$ has been used to obtain values for the parameters $\varepsilon(-2)$ and $\theta_{D}(-2)$. 


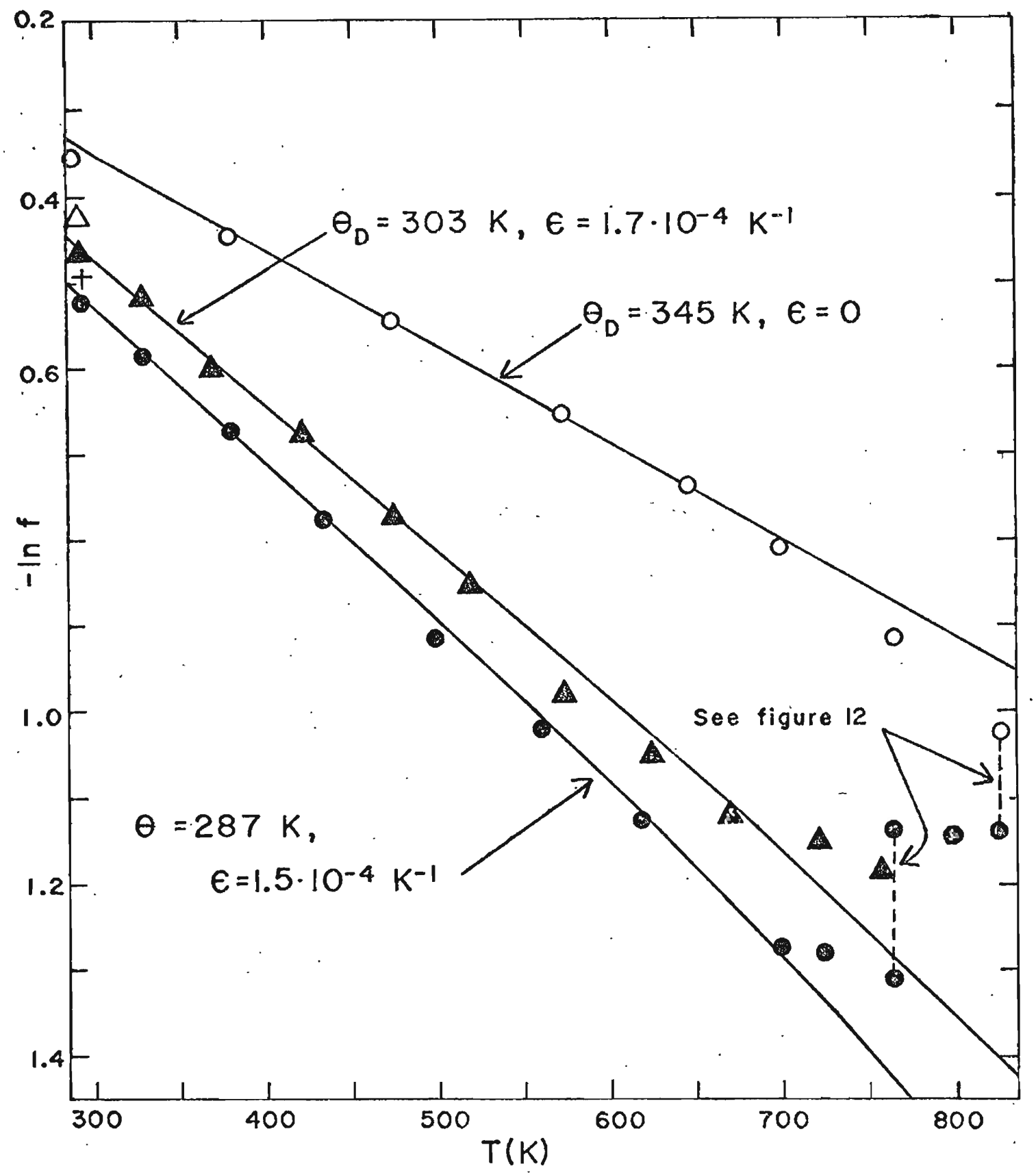

Figure 11. Temperature dependence of $(-$ in $f)$ for $A u l l l(\Delta, \Delta)$, $A u I I I B(+)$, and $A u I V(0,0)$. The solid symbols indicate that the source temperature was being increased between measurements; the open symbols indicote that the source temperature was being decreased befween meosurements. The solid lines indicate the expected behovior, equation (23). 


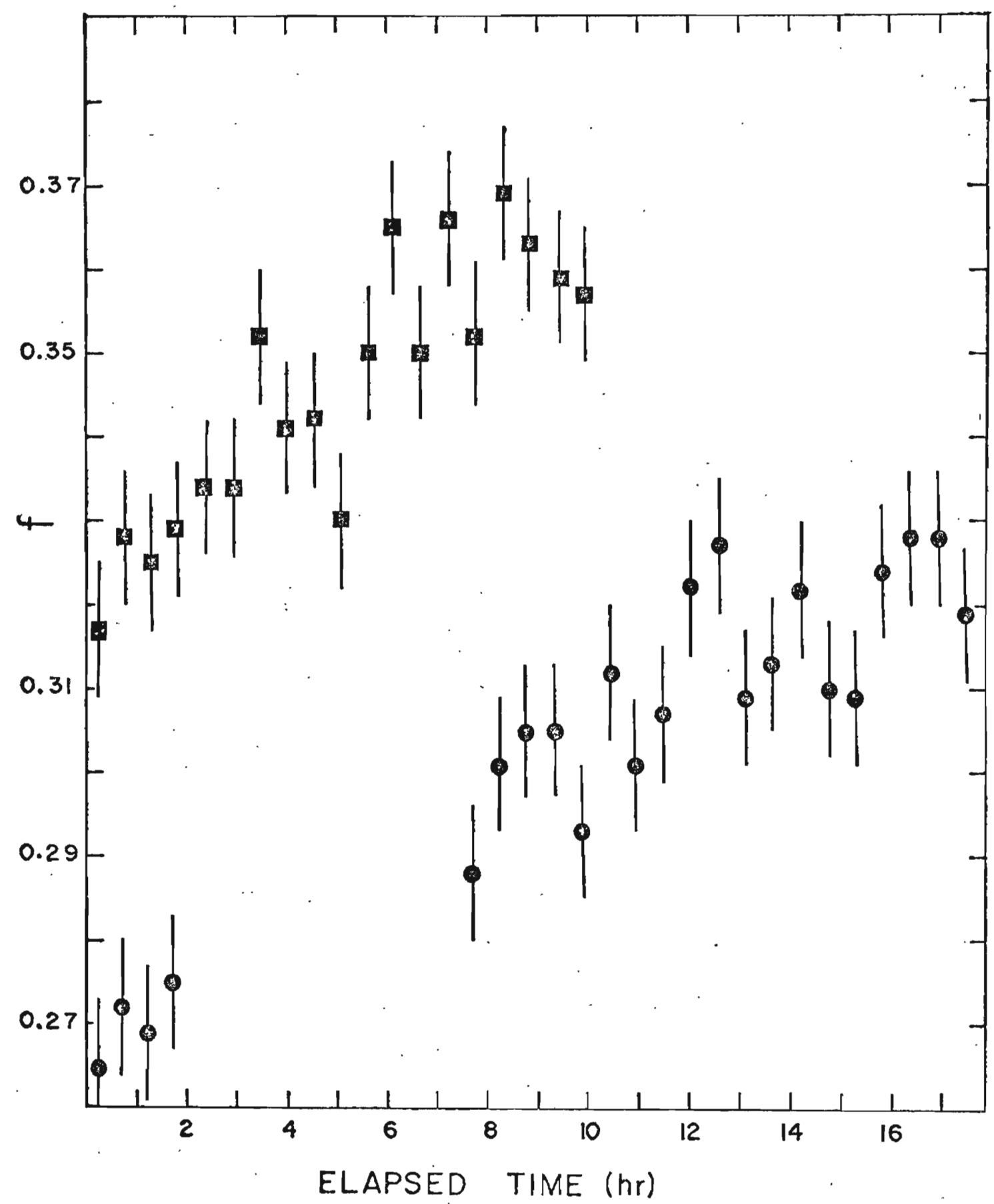

Figure 12. Time dependence of the Debye-Waller factor of Au IV at $765 K(0)$ and $829 K(\mathbb{R})$. 
site (site B) which has a smaller mean squared displacement. These two sites are discussed below.

\section{SUBSTITUTIONAL SITE}

Lattice site A has been populated by quenching the sources from a relatively high temperature ( $1273 \mathrm{~K}$. in the case of Au IV). In the following section this site i.s interpreted, on the basis of the line. shape, to be a substitutional site. The anharmonicity and strength of the force constants binding the impurity in this site are also discussed.

The line shape obtained from $\mathrm{Fe}^{57}$ atoms in site $\mathrm{A}$ is shown in figure 7:; the line shape parameters are summarized in table IV. For both $\mathrm{Au}$ III and $\mathrm{Au}$ IV, the measured line width is broader than natural width. Similarly, for the cases of $\mathrm{Fe}^{57}$ in $\mathrm{Pd}$ and $\mathrm{Pt}{ }^{15}$ we have been unable to prepare sources having natural width lines. The broadening apppears to be intrinsic, and not a result of the method used to prepare the sources since two different techniques of source preparation have been used; The $\mathrm{Co}^{57}$, in the form of $\mathrm{CoCl}_{2}$, was pipetted onto the surface of the Pd and Pt sources while electrolytic deposition was used to prepare the Au sources. Furthermore, natural line width $\mathrm{Cu}$ sources have been prepared using both techniques.

Impurity atoms which differ in size from the host atoms tend to segregate at dislocations where they can help to relieve the strain field of the dislocation ${ }^{32}$. The local symmetry of the lattice in the dislocation is less than cubic; this noncubic symmetry will give rise to an electric field gradient at the site of impurity atoms which have 
TABLE IV

LINE SHAPE PARAMETERS OF Fe 57 IN Au FOR QUENCHED AND ANNEALED SOURCES

\begin{tabular}{|c|c|c|c|}
\hline $\begin{array}{c}\text { Heat } \\
\text { treatment }\end{array}$ & $\begin{array}{c}\text { Iine } \\
\text { wid.th } \\
(\mathrm{mm} / \mathrm{sec})\end{array}$ & $\begin{array}{c}\text { Chemical } \\
\text { shift } \\
(\mathrm{mm} / \mathrm{sec})\end{array}$ & $\begin{array}{c}\text { Quadrupole } \\
\text { splitting } \\
\text { (mm/sec) }\end{array}$ \\
\hline $\begin{array}{c}\text { Quenched } \\
\text { Annealed }\end{array}$ & $\begin{array}{c}0.235 \pm .005 \\
0.40 \pm .02\end{array}$ & $\begin{array}{c}-0.635 \pm .005 \\
-0.34 \pm .01\end{array}$ & $0.40 \pm .01$ \\
\hline
\end{tabular}


segregated at dislocations. The resulting line shape will be a superposition of the quadrupole split doublet from impurities in dislocations and the single line from impurities in substitutional lattice sites; it may appear to be a single broadened line.

Further support for the above conclusion can be obtained by comparing the magnitude of the lattice spacing of the host lattice with the observed line broadening of the sources mentioned above; this comparison is made in table $V$. The correlation between the increased Iine width and the increase in the lattice spacing can be understood as follows: As the size difference between the host and impurity atoms increases, the tendency towards segregation at dislocations will increase 32 ; this will increase the contribution of the quadrupole split doublet to the composit spectrum and result in a broader line.

Based on the above interpretation of the line shape of the quenched Au sources, it is concluded that site A is a substitutional lattice site.

The next step in the analysis of the data is to obtain values for the characteristic temperature $\theta_{D}(-2)$ and the anharmonicity parameter $\varepsilon(-2)$ for $\mathrm{Fe}^{57}$ impurities in the substitutional site. Before this can be done however, the following points must be considered:

1) Clearly, above $700 \mathrm{~K}$ a significant fraction of the Co atoms have diffused from the substitutional site into site $B$, as is evidenced by the anomalous increase in $\mathrm{f}$. Therefore, the data above $700 \mathrm{~K}$ cannot be used to determine $\theta_{D}(-2)$ and $\varepsilon(-2)$. Below $700 \mathrm{~K}$ it is difficult to determine what fraction of the impurities 


\section{TABLE V}

COMPARISON OF LINE BROADENING WITH THE LATTICE

PARAMETER OF THE HOST LATTICE FOR

$\mathrm{Pd}, \mathrm{Pt}$, and $\mathrm{Au}$ SOURCES

s.

\begin{tabular}{|l|l|l|}
\hline Source & \multicolumn{1}{|c|}{$\begin{array}{c}\text { Line width } \\
(\mathrm{mm} / \mathrm{sec})\end{array}$} & $\begin{array}{c}\text { Lattice spacing } a \\
(\AA)\end{array}$ \\
\hline Pd & $0.21 \mathrm{~b}$ & 3.88 \\
Pt & $0.21 \mathrm{~b}$ & 3.92 \\
Au & 0.235 & 4.07 \\
\hline
\end{tabular}

(a) These values were taken from reference 34.

(b) These values were taken from reference 15. 
have diffused into site $B$. A small amount of diffusion of the Co while the measurements below $700 \mathrm{~K}$ were being made would not be noticed, but could reduce the curvature of the measured $f$ values making $\varepsilon(-2)$ appear smaller than it really is.

2) The values of $\theta_{D}(-2)$ obtained from the $f$ measurements made on Au III is greater than the value obtained from the measurements made on Au IV. Obviously, some of the Fe atoms in Au III were in site B. It therefore appears Iikely that some of the $\mathrm{Fe}$ atoms in $\mathrm{Au}$ IV are also in site $B$. If this is the case, the value of $\theta_{D}(-2)$ obtained from the measurements made on $\mathrm{Au}$ IV will be greater than the actual characteristic temperature of the substitutional site. If only a few percent of the impurities are in site $B$, the discrepancy between the measured value and the actual value of $\theta_{D}(-2)$ for the substitutional site will be within the experimental uncertainty.

Keeping these difficulties in mind we can tentatively assign values to $\left[\theta_{D}(-2)\right]_{i m p}$ and $[\varepsilon(-2)]_{i m p}$ for the substitutional site. These values are given in table VI and were obtained from the solid lines in figure 11.

There is no experimental determination of $[\varepsilon(-2)]$ host for $\mathrm{Au}$ from neutron scattering experiments. Therefore, values for $[\varepsilon(-2)]_{\text {host }}$ must be obtained from equations (24) and (31). Pathak and Deo ${ }^{14}$ have made these calculations; their results are included in table VI. 
TABLE VI

IATTICE DYNAMICAI PARAMETERS FOR Fe 57

IN AU FOR THE SUBSTITUTIONAL

LATTICE SITE

\begin{tabular}{|c|c|c|c|c|}
\hline $\begin{array}{c}{[\varepsilon(-2)]_{\text {imp }}} \\
(/ K)\end{array}$ & $\begin{array}{c}{[\varepsilon(-2)]_{\text {host }}{ }^{a}} \\
(/ K)\end{array}$ & $\begin{array}{c}{[\varepsilon(-2)]_{\text {host }}{ }^{b}} \\
(/ K)\end{array}$ & $\begin{array}{c}{\left[\theta_{D}(-3)\right]_{\text {host }}{ }^{c}} \\
(K)\end{array}$ & $\alpha$ / ' ' \\
\hline $1.5 \cdot 10^{-4}$ & $0.6 \cdot 10^{-4}$ & $2.5 \cdot 10^{-4}$ & 164 & 1 \\
\hline
\end{tabular}

(a) From equation (24).

(b) From equation (31).

(c) This value was obtained from reference 10 . 
Maradudin and Flinn ${ }^{35}$ have considered a harmonic face centered cubic lattice with one central force constant and nearest neighbor interactions only: They find that

$$
\begin{aligned}
& {\left.\left[\theta_{D}(-2)\right]_{i m p}=q^{-\frac{1}{2}\left[\theta_{D}\right.}(-2)\right]_{h o s t} \cdot\left[1+0.60\left|\frac{\alpha-\alpha}{\alpha}\right|\right.} \\
&\left.+0.74\left(\frac{\alpha-\alpha}{\alpha}\right)^{2}+\ldots\right]^{-\frac{1}{2}}
\end{aligned}
$$

where $q$ is the mass ratio $\mathrm{m}_{\text {imp }} / \mathrm{m}_{\text {host }}$ and $\alpha$ and $\alpha '$ are respectively the host and the impurity force constants. The resulting quadratic equation in $\alpha / \alpha^{\prime}$ can be solved if

$$
q^{-\frac{1}{2}} \frac{\left[\theta_{D}(-2)\right]_{\text {imp }}}{\left[\theta_{D}(-2)\right]_{\text {host }}}<1.07
$$

For relative force constant changes $\left(\alpha-\alpha^{\prime}\right) / \alpha>0.16$ higher order terms may become important. $\left[\theta_{D}(-2)\right]_{\text {host }}$ was obtained from heat capacity measurements by assuming that the ratio of the $(-2)$ moment to the $(-3)$ moment is $0.93^{15}$. Values for $\left[\theta_{D}(-3)\right]_{\text {host }}$ and $\alpha / \alpha^{\prime}$ are given in table VI.

From table VI it can be seen that $\mathrm{Fe}$ in $\mathrm{Au}$ (in the substitutional lattice site) is about as anharmonic as Au in Au. Also, the force constant holding the $F e$ atom in the substitutional site is the same as the force constant holding a Au atom in the lattice.

\section{INTERSTITIAI SITE}

Site B has been formed by annealing the Au sources at intermediate temperatures $(700$ to $800 \mathrm{~K})$. It is shown below that this site is an interstitial lattice site. The data is shown to be consistent with the interpretation that the interstitial is a split $\langle 100\rangle$ or dumbbell 
interstitial formed by substituting a pair of Co impurity atoms (or, since $F e$ atoms are also present in the source, an Fe-Co pair) for one of the Au atoms.

The characteristics of lattice site $B$ are:

1) Noncubic symmetry - A line shape measurement was made on Au IV after the completion of the $f$ measurements; it is shown in figure 13. The major contribution (about 75\%) to the spectrum is from a pair of broad lines arising from the nuclear quadrupole interaction. The remainder of the spectrum is due to Fe atoms in the substitutional site. The existence of the nuclear quadrupole interaction indicates that the symmetry of site $B$ is less than cubic.

2) Increased s-electron density - The chemical shift of the quadrupole split doublet $(-0.34 \mathrm{~mm} / \mathrm{sec})$ is less - negative than the chemical shift of the singlet $(-0.635$ $\mathrm{mm} / \mathrm{sec})$. This indicates that the source emission line for impurities in site $B$ is shifted toward lower energy than the source line for impurities in the substitutional site, and hence the s-electron density at site $B$ is greater.

3) Tight binding - After annealing at $829 \mathrm{~K}$, Au IV was cooled down; f measurements were made on the source as it cooled, see figure 11. The large value of $\theta_{D}(-2)$ which was obtained from the data and the corresponding reduction in the mean squared displacement require that 


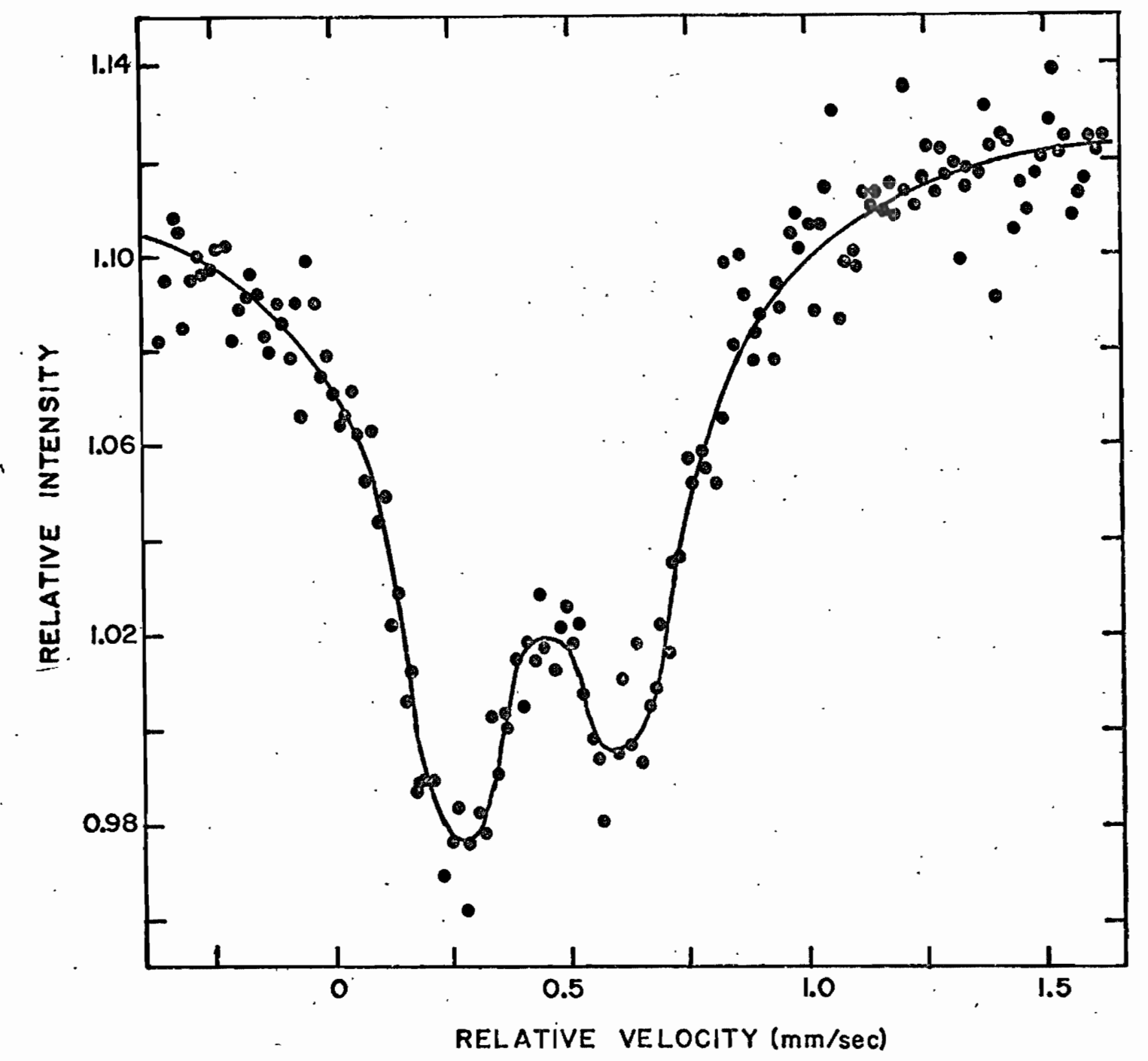

Figure 13. Line shape of AuIV taken on the speaker drive using one of the lines of the lines of a nitroprusside absorber; the velocity scale has been adjusted to give velocities relative to an iron absorber. The spectrum was taken after the completion of the measurements. The asymmetry of the quadrupole split doublet is due to approximately $25 \%$ of the Fe impurities which are still in substitutional sites. 
impurity atoms in the substitutional site are bound by woaker force constants than impurities in site $B$.

In an interstitial site the impurity is crowded by the surroundIng atoms of the crystal, resulting in a decreased mean squared displacement and an increase in the s-electron density. Also, the symmetry of the interstice will be less than cubic, resulting in a quadrupole split emission line. Site $B$ is therefore characteristic of an interstitial site.

The long time required for the formation of the interstitial site (see figure 12) can be explained only if diffusion is the mechanism which transports the impurity from one lattice site into the other. Diffusion will also explain why the characteristic time required to Iorm the interstitial site decreases with increasing temperature.

Site B cannot be an ordinary interstitial; i.e. one in which the impurity is surrounded by host atoms occupying substitutional lattice sites. If it were, the time required for the formation of the interstitial would be comparable to the time it takes the impurity to move from one lattice site to another; this time is about $10^{-5} \mathrm{sec}$ for Co diffusing in $\mathrm{Au}$ at $800 \mathrm{~K}{ }^{36}$. We therefore propose that the impurities are in split 100 , or dumbbell, interstitial sites. From figure 14 it can be seen that the impurities in the dumbbell site occupy interstices. The formation of the dumbbell depends on diffusion in the following manner: Initially the impurities are in substitutional lattice sites having a mean separation of about 12 lattice spacings. In order to form the dumbbell interstitial two impurities must become nearest neighbors; this process occurs by diffusion. After becoming 


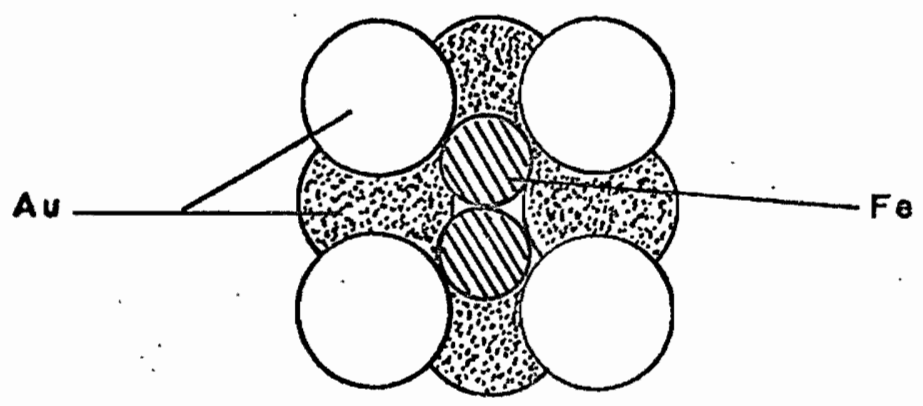

Figure 14. Position of the impurity dumbbell in the Au lattice. The symmetry axis of the dumbbell can lie along any of the three [100] directions. 
nearest neighbors the impurities move into the same lattice site and release a vacancy. The energy gained from the formation of this vacancy makes up for the energy required to form the dumbbell. Since a vacancy is formed at the same time that the dumbbell is formed, it is probable that an equilibrium exists between the relative number of dumbelis and the vacancy concentration. The equilibrium would favor dumbbells at low temperatures where the vacancy concentration is low. At higher temperatures, where the vacancy concentration is larger, fewer of the impurities will be found in the dumbbell site. Near the melting point of Au nearly all of the impurity atoms should be in the substitutional lattice site.

Estimates of the force constant ratio $\alpha / \alpha$, and the anharmonicity parameter for $\mathrm{Fe}$ in $\mathrm{Au}$ in the interstitial site have not been included in table VI. Too many of the Fe atoms in $\mathrm{Au}$ IV are still in the substitutional site for a reasonable estimate of these parameters to be made. 
SUMMARY AND CONCLUSION

Precision measurements of the Debye-Waller factor of $\mathrm{Fe}^{57}$ in $\mathrm{Au}$ using the wide black absorber technique have been made in the tempera-, ture range from room temperature to $830 \mathrm{~K}$. The major source of error arises from the $\mathrm{Au} \mathrm{I} \mathrm{L}_{\gamma} \mathrm{x}$-rays which are unresolved by the radiation detection system from the $14.4 \mathrm{keV}$ gamma ray. The $\mathrm{K}$ absorption edge of $\mathrm{Br}$ has been used to correct for the presence of the $\mathrm{x}$-rays.

These measurements have revealed the existence of two nonequivalent lattice sites which the $\mathrm{Co}^{57}$ impurity (parent of $\mathrm{Fe}^{57}$ ) may occupy. One of the sites has been populated by quenching the source from temperatures greater than $1150 \mathrm{~K}$ and has been identified as a substitutional lattice site. From an analysis of the Mössbauer spectrum it is concluded that some of the substitutional sites may be in the vicinity of dislocations. It has been determined that the impurity-host force constant for $\mathrm{Fe}^{57}$ atoms in the substitutional site is about the same as the host-host force constant; the experimental value of the anharmonicity parameter $\varepsilon(-2)$ for $\mathrm{Fe}^{57}$ in $\mathrm{Au}$ is nearly the same as calculated values of $\varepsilon(-2)$ for pure Au.

The second site forms very slowly upon annealing the source at intermediate temperatures and has been identified as being interstitial in character. The slow rate of formation of the interstitial site 


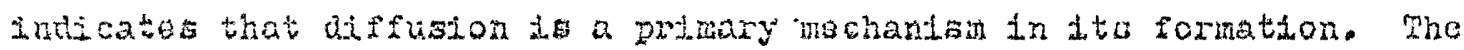

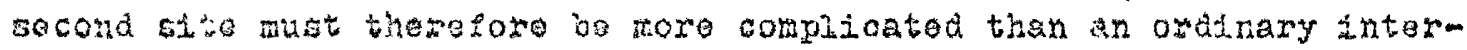
stutin. It is proposed that this sate is a split $\langle 100\rangle$, or dumbeli, interstitial formed by substituting two impurity atoms for one of the Au atoms.

It was pointed out in the introduction that previous measurements ${ }^{1-5}$ are in disagreement with each other. Since none of these investigations has revealed the existence of the interstitial lattice site at least part of the disagreement must be attributed to the existence of this site.

In additson to establishing the extetonce of two nonequivalent lattice sites for $C^{57}$ in Au, the present Investigation has also shown that for some cases precision i measurements can be used for studying both bulk diffusion of impurities and the metallurgy of alloys. Until now, the usefulness of $f$ measurements for this purpose has been generally overlooked. 


\section{REFERENCES}

1. R. M. Housley, F. Hess and T. Sinnema, Solid State Comm. $\underline{6}, 375$ (1968).

2. R. C. Knauer and J. G. Mullen, Appl. Phys. Letters 13, 150 (1968).

3. V. I. Nikolaev and S. S. Yakimov, Soviet Phys. JETP 19, 264 (1964).

4. D. L. Sprague, PhD Thesis, University of Washington, 1967 (unpublished).

5. W. A. Steyert and R. D. Taylox, Phys. Rev. 134, A716 (1964).

6. H. J. Lipkin, Ann. Phys. 2, 332 (1960).

7. A. J. F. Boyle and N. E. Hall, Rept. Progr. Phys. 25, 441 (1962).

8. A. A. Maradudin and P. A. Flinn, Phys. Rev. 129, 2529 (1963).

9. R. M. Housley and F. Hess, Phys. Rev. 146, 517 (1966).

10. C. Kittel, "Introduction to Solid State Physics," (J. Wiley and Sons, N. Y., 1966).

11. G. W. Lehman and R. E. DeWames, Phys. Rev. 131, 1008 (1963).

12. N. F. Mott and H. Jones, "The Theory of the Properties of Metals and Alloys," (Dover, N. Y., 1958).

13. A. H. Muir, "Tables and Graphs for Computing Debye-Waller Factors in Mössbauer Effect Studies," (Atomic International Doc. AI-6699, 1962).

14. M. Blackman, Enc. of Phys. 2, 325 (1955).

15. R. H. Nussbaum, D. G. Howard, W. I. Nees and C. F. Steen, Phys. Rev. 123, 653 (1968).

16. J. G. Dash, D. P. Johnson, and W. M. Visscher, Phys. Rev. 173, 653 (1968). 
17. H. J. Lipkin, Ann. Phys. 26, 115 (1964).

18. T. H. K. Barron, W. T. Berg and J. A. Morrison, Proc. Roy. Soc. (London) A242, 478 (1957).

19. K. N. Pathak and B. Deo, Physica 35, 167 (1967).

20. H. Frauenfelder, "The Móssbauer Effect," (W. $\Lambda$. Benjamin, N. Y., 1963).

21. Gol'Danskii, "The Mössbauer Effect and Its Applications in Chemistry," (Consultants Bureau, N. Y., 1964).

22. W. I. Nees, M. S. Thesis, Portland State University, 1968 (unpublished).

23. R. H. Nussbaum, F. Gerstenfeld, and J. K. Richardson, Ammer. J. Phys. 34, 40 (1966).

24. J. Stephen, Nucl. Inst. and Meth. 26, 269 (1965).

25. I. Dèzsi and B. Molnars Nucl. Inst. and Meth. 24, 105 (1967).

26. A. Mustachi, Nucl. Inst, and Meth. 26, 219 (1964).

27. S. M. Qaim, P. J. Black and M. J. Evans, Journal of Phys. C (Proc. Phys. Soc.) 2 1, 1388 (1968).

28. R. M. Housley, N. E. Erickson and J. G. Dash, Nucl. Inst. and Meth. 27, 29 (1964).

29. E. Storm and H. I. Isreal, Nucl. Data Tables Sec. A 2, 1 (1970).

30. J. H. Scofield, Phys. Rev. 179, 9 (1969).

31. G. C. Nelson and B. G. Saunders, Phys. Rev. 188; 108 (1969).

32. R. M. Housley, Nucl. Inst. and Meth. 25, 77 (1965).

33. R. W. Cahn, "Physical Metallurgy," (J. Wiley and Sons, N. Y., 1965).

34. W. B. Pearson, "Handbook of Lattice Spacings and Structure of Metals and Alloys," (Pergamon Press, Ita., London, 1964).

35. A. A. Maradudin and P. A. Flinn, Phys. Rev. 126, 2059 (1962).

36. D. Duhl, K. Hirano and M. Cohen, Acta Met. 11, 1 (1963). 